\title{
Polynomially convex embeddings of odd-dimensional closed manifolds
}

\author{
By Purvi Gupta at Bangalore and Rasul Shafikov at London, ON
}

\begin{abstract}
It is shown that any smooth closed orientable manifold of dimension $2 k+1$, $k \geq 2$, admits a smooth polynomially convex embedding into $\mathbb{C}^{3 k}$. This improves by 1 the previously known lower bound of $3 k+1$ on the possible ambient complex dimension for such embeddings (which is sharp when $k=1$ ). It is further shown that the embeddings produced have the property that all continuous functions on the image can be uniformly approximated by holomorphic polynomials. Lastly, the same technique is modified to construct embeddings whose images have nontrivial hulls containing no nontrivial analytic disks. The distinguishing feature of this dimensional setting is the appearance of nonisolated CR-singularities, which cannot be tackled using only local analytic methods (as done in earlier results of this kind), and a topological approach is required.
\end{abstract}

\section{Introduction}

The problem of finding the least Euclidean dimension into which all abstract manifolds of a fixed dimension admit embeddings with certain prescribed properties appears in many different contexts in geometry, as exemplified by the classical results of Nash, Grauert-Morrey, Remmert-Bishop-Narasimhan, etc. In a similar spirit, the topological consequences of imposing certain convexity-type conditions on manifolds in $\mathbb{C}^{n}$ has been a recurrent topic of interest in complex analysis; for instance, see $[2,8,14,27]$. Along these lines, we study the polynomially convex embedding problem: what is the least $n$ such that all closed smooth real $m$-manifolds admit polynomially convex smooth embeddings into $\mathbb{C}^{n}$ ?

Main result. We prove the following result in this paper.

Theorem 1.1. Let $M$ be a closed orientable smooth manifold of real dimension $2 k+1$, where $k \geq 2$. Then there is a smooth embedding $\iota: M \hookrightarrow \mathbb{C}^{3 k}$ such that

(1) $M^{\prime}=\iota(M)$ is totally real except along a finite union of simple closed real curves in $M^{\prime}$,

The corresponding author is Purvi Gupta.

Purvi Gupta is supported in part by a UGC CAS-II grant (Grant No. F.510/25/CAS-II/2018(SAP-I)). Rasul Shafikov is partially supported by the Natural Sciences and Engineering Research Council of Canada. 
(2) $M^{\prime}$ is a polynomially convex subset of $\mathbb{C}^{3 k}$, and

(3) any continuous function on $M^{\prime}$ can be uniformly approximated by holomorphic polynomials on $M^{\prime}$.

A compact set $K \subset \mathbb{C}^{n}$ is polynomially convex if its polynomially convex hull, defined as

$$
\widehat{K}=\left\{z \in \mathbb{C}^{n}:|P(z)| \leq \sup _{K}|P| \text { for all holomorphic polynomials } P \text { on } \mathbb{C}^{n}\right\},
$$

coincides with $K$. Embeddability of manifolds as polynomially convex compacts in $\mathbb{C}^{n}$ is important in view of the Oka-Weil theorem: if $K=\widehat{K}$, then any function holomorphic on some neighborhood of $K$ can be uniformly approximated on $K$ by holomorphic polynomials. A partial converse also holds: if all continuous functions on $K$ can be uniformly approximated on $K$ by holomorphic polynomials, then $K=\widehat{K}$. Thus, (3) implies (2) in Theorem 1.1. However, the polynomial embeddability of $M$ is the crux of the matter, and poses the main technical challenge.

Until recently, the best known bound on the optimal embedding dimension for polynomially convex embeddings was the same as that for totally real embeddings, i.e., where the image admits no complex lines in any of its tangent planes. These two types of embeddings are related by the result that any $m$-dimensional totally real submanifold in $\mathbb{C}^{n}, m<n$, can be made polynomially convex after a small perturbation; see [15], [13] and [25]. It is known that if $n \geq\left\lfloor\frac{3 m}{2}\right\rfloor$, then any closed $m$-dimensional manifold can be embedded into $\mathbb{C}^{n}$ as a totally real submanifold. This bound is sharp for totally real embeddability (see [21]), that is, if $n<\left\lfloor\frac{3 m}{2}\right\rfloor$ then one cannot always avoid $C R$-singularities, i.e., points where the tangent plane contains nontrivial complex subspaces. When $m \leq 3$, this bound is also sharp for polynomially-convex embeddability as no real $n$-dimensional submanifold in $\mathbb{C}^{n}$ can be polynomially convex, see [30, Section 2.3]. However, when $m \geq 4$, the extent to which the bound for polynomially convex embeddability can be improved is not known.

In our earlier paper [18], this bound was improved by one for even-dimensional manifolds. That is, for every $k \geq 2,(2 k)$-dimensional manifolds admit polynomially convex smooth embeddings into $\mathbb{C}^{3 k-1}$. Here, generic CR-singularities are isolated points. As in the case of totally real embeddings, the method of small perturbations works in this setting, with some additional local analysis required near the singularities. Theorem 1.1 extends this improvement of bound to all odd-dimensional manifolds of dimension at least 5, under the assumption of orientability. In this setting, CR-singularities generically form closed real curves on the manifold. While isolated CR-singularities are well-studied in all relevant dimensions, starting from the seminal work [7], the literature on the global properties of CR-singular sets of positive dimension is rather sparse; see [11] and [32] for some global results. Such sets carry nontrivial topology whose properties are not very well understood. Thus, to obtain embeddings with prescribed convexity properties, one can no longer rely on local analysis alone and must use topological methods.

Idea of the proof. We briefly describe the construction that yields part (2) of Theorem 1.1. First, by a standard argument, we observe that a generic embedding of $M$ is totally real except along a finite union of disjoint simple closed curves of CR-singularities. A tubular neighborhood in the embedded manifold of such a curve is an instance of a tube enclosing a CR-singular curve in $\mathbb{C}^{3 k}$ (see Definition 4.1). We call two such disjoint tubes totally real 
cylindrically (TRC) cobordant if their boundaries can be joined by a totally real cylindrical manifold within $\mathbb{C}^{3 k}$. Using a relative $h$-principle, we show that the TRC cobordism class of a (parametrized) tube is determined by the homotopy class of its frame map - a map from $S^{1} \times S^{2 k-1}$ into the complex Stiefel manifold $W_{2 k+1,3 k}$ induced by a field of frames on the given tube, see (4.1). From this, we deduce that the possible homotopy classes of all such maps can be enumerated by $\mathbb{Z}$ when $k$ is even, and by $\mathbb{Z} \oplus \mathbb{Z}_{2}$ when $k$ is odd. We then construct models of tubes (enclosing CR-singular curves) that realize all the possible enumerations, and satisfy the crucial property that they are polynomially convex. When $k$ is even, the construction is a straightforward modification of the Beloshapka-Coffman normal form discussed in Section 2.2. However, when $k$ is odd, this construction has to be adjusted using a Hopf fibration to account for the torsion that appears in this setting. To summarize, a small neighborhood of any CR-singular curve of a generic embedding of $M$ is TRC cobordant to one of the constructed polynomially convex models. Effectively, this gives the following procedure: for each CR-singular curve in the (embedded) manifold, we cut out such a tubular neighborhood, and glue instead a TRC cobordant polynomially convex tube using a cylindrical manifold that joins their boundaries. Finally, using the fact that the CR-singular set of the new embedding admits a polynomially convex neighborhood in the manifold, we perform a small perturbation (using [5]) to make the manifold globally polynomially convex.

Part (3) of Theorem 1.1 now follows from a combination of three classical approximation results, including the aforementioned result due to Oka-Weil. This part of the theorem can be paraphrased as follows: for any closed $(2 k+1)$-manifold $M$ as in Theorem 1.1, there exist $3 k$ smooth functions on $M$ that generate $C(M)$, the algebra of continuous complex-valued functions on $M$.

Scope for further improvements. There are several questions that remain open in the context of Theorem 1.1. First, the assumption of orientability may simply be an artefact of our proof. In the absence of orientability, one will have to account for "nonorientable tubes" enclosing CR-singular curves, but the broader technique still holds promise. Second, while there are examples of closed $m$-dimensional real manifolds, $m \geq 2$, that cannot be embedded into $\mathbb{C}^{n}$ when $n=\left\lfloor\frac{3 m}{2}\right\rfloor-1$, see [21], all the known examples are nonorientable when $m=2 k+1$ and $k$ is odd, and it is not clear whether $n=\left\lfloor\frac{3 m}{2}\right\rfloor$ is sharp for orientable manifolds in this case. We note that totally real embeddability would give an easier proof of (2) and a stronger version of (3) in Theorem 1.1. Finally, in the case of isolated CR-singularities, any embedding of a compact manifold with boundary can be perturbed to be totally real and polynomially convex (see [18]). It is not clear whether our proof can be modified to obtain totally real polynomially convex embeddings of $(2 k+1)$-dimensional manifolds with boundary in $\mathbb{C}^{3 k}$.

Whether the polynomially convex embedding dimension can be improved further than $\left\lfloor\frac{3 m}{2}\right\rfloor-1$, for $m \geq 6$, remains an open problem. If one only seeks topological embeddings, the sharp bound is known from [31], wherein polynomially convex topological embeddings of all $m$-dimensional smooth manifolds (in fact, even simplicial polytopes) into $\mathbb{C}^{m+1}$ have been constructed. However, these embeddings are highly nonsmooth. For smooth embeddings, the next possible dimensional improvement already poses several technical difficulties. One has to reckon with surfaces of CR-singularities. This causes some difficulty in both enumerating all the topological possibilities (in the TRC cobordism sense) and constructing polynomially convex models. Further, while the Beloshapka-Coffman normal form is still available, the nondegeneracy required to invoke this form cannot be guaranteed everywhere as degenerate 
CR-singular points generically form a set of codimension two in the manifold, and can no longer be separated from the CR-singular set by a simple transversality argument.

Embeddings with no analytic disks in their hulls. The technology developed to prove Theorem 1.1 can be used to lower the bound for another embedding problem (raised in [23]): what is the least $n^{\prime}$ such that every compact m-dimensional smooth manifold can be smoothly embedded into $\mathbb{C}^{n^{\prime}}$ as some $\Sigma$ with $\widehat{\Sigma} \backslash \Sigma$ nonempty but containing no analytic disk, i.e., there is no nonconstant holomorphic map from the unit disk into $\widehat{\Sigma} \backslash \Sigma$ ? This can also be asked for rational hulls where, if $K \subset \mathbb{C}^{n}$ is a compact set, its rational hull is

$$
h_{r}(K)=\left\{z \in \mathbb{C}^{n}: p(z) \in p(K) \text { for all holomorphic polynomials } P \text { on } \mathbb{C}^{n}\right\} .
$$

Part of the motivation for this problem comes from the fact that all the classical constructions of nontrivial hulls with no analytic disks involve highly nonsmooth sets. For smooth embeddings, the best known bound for surfaces is $n^{\prime} \leq 3$, obtained in [23] via explicit embeddings. For higher dimensions, $n^{\prime} \leq\left\lfloor\frac{3 m}{2}\right\rfloor-1$ when $m$ is even, as seen in [18], and $n^{\prime} \leq\left\lfloor\frac{3 m}{2}\right\rfloor$ when $m$ is odd, as proved in [5]. For orientable manifolds, we improve the latter as follows.

Theorem 1.2. Given any closed orientable smooth manifold $M$ of real dimension $2 k+1$, $k \geq 2$, there is a smooth embedding of $M$ into $\mathbb{C}^{3 k}$ with image $\Sigma$ so that $\widehat{\Sigma} \backslash \Sigma$ is nonempty but contains no analytic disk, and $\widehat{\Sigma}=h_{r}(\Sigma)$.

Structure of the paper. In Section 2, we collect some basic facts about CR-singularities that allow us to make certain simplifying assumptions in our proofs. The Beloshapka-Coffman normal form, in particular, is discussed in Section 2.2. In Section 3, we lay the groundwork for the topological aspects of the proofs. After establishing some essential notation in Section 3.1, we explicitly compute generators of certain homotopy groups associated with the Stiefel manifold $W_{2 k+1,3 k}$ in Sections 3.2 and 3.3. Section 4 is devoted to the study of tubular neighborhoods of certain curves in $(2 k+1)$-dimensional submanifolds of $\mathbb{C}^{3 k}$. For such neighborhoods, we introduce a topological invariant (index) in Section 4.1, and an equivalence relation (totally real cylindrical cobordism) in Section 4.2. We construct polynomially convex representatives of all possible equivalence classes in Section 4.3. Finally, the proofs of the main results are carried out in Section 5.

\section{CR-geometric preliminaries}

We recall some facts about CR-singularities of real $m$-dimensional submanifolds in $\mathbb{C}^{n}$. Putting together these facts, we obtain part (1) of Theorem 1.1.

2.1. CR-singularities. The $C R$-dimension of a real submanifold $M \subset \mathbb{C}^{n}$ at a point $p \in M$ is the (complex) dimension of the maximal complex linear subspace $T_{p}^{c} M$ of $T_{p} M$, the tangent plane of $M$ at $p$ (considered as a subset of $\mathbb{C}^{n}$ ). If $\operatorname{dim} M \leq n$ and $M$ is in general position, then it will be generically totally real, i.e., the CR-dimension of $M$ will be 0 almost everywhere. In this case, a point $p \in M$ is called $C R$-singular if $\operatorname{dim}_{\mathbb{C}} T_{p}^{c} M \geq 1$. Furthermore, we call $p \in M$ a $C R$-singularity of order $\mu$ if $\operatorname{dim}_{\mathbb{C}} T_{p}^{c} M=\mu$. Given $\mu \in \mathbb{N}_{0}$, if $S_{\mu}$ denotes the set of CR-singular points of $M$ of order $\mu$, then $S_{\mu}$ is either empty or is a (not necessarily 
closed) submanifold of $M$ of dimension $m-\left(2 \mu^{2}+2 \mu(n-m)\right)$ and

$$
\overline{S_{\mu}}=\bigcup_{\nu \geq \mu} S_{\nu},
$$

see [11] for more details. From this it follows that if $n \geq\left\lfloor\frac{3 m}{2}\right\rfloor$, then any $m$-dimensional manifold admits a totally real embedding into $\mathbb{C}^{n}$. If $m=2 k$ and $n=3 k-1$, then all the CR-singularities of a generic $M$ are isolated points, while if $m=2 k+1$ and $n=3 k$, then a generic $M$ has a one-dimensional set of CR-singularities of order 1 and no CR-singularities of order 2 or higher. Thus, we have

Lemma 2.1. Suppose that $\iota: M \hookrightarrow \mathbb{C}^{3 k}$ is a generic smooth embedding of a smooth $(2 k+1)$-dimensional closed manifold. Then the set of $C R$-singularities of $\iota(M)$ is a finite union of smooth simple closed real curves.

2.2. The Beloshapka-Coffman normal form. Building on Beloshapka's work [6] for $n=5$, Coffman introduced in [9] a notion of nondegeneracy for a CR-singular point of an $m$-dimensional manifold $M$ in $\mathbb{C}^{n}$, where $\frac{2}{3}(n+1) \leq m<n, n \geq 5$. He showed that near a nondegenerate CR-singular point, $M$ is locally formally equivalent to the Beloshapka-Coffman normal form, which is given by

$$
\begin{aligned}
\mathcal{B}^{m, n}=\left\{\left(z_{1}, \ldots, z_{n}\right) \in \mathbb{C}^{n}:\right. & \operatorname{Im} z_{j}=0,2 \leq j \leq m-1, \\
& z_{m}={\overline{z_{1}}}^{2}, \\
& z_{m+1}=\left|z_{1}\right|^{2}+\overline{z_{1}}\left(\operatorname{Re} z_{2}+i \operatorname{Re} z_{3}\right), \\
& z_{\ell}=\overline{z_{1}}\left(\operatorname{Re} z_{2(\ell-m)}+i \operatorname{Re} z_{2(\ell-m)+1}\right), \\
& m+2 \leq \ell \leq n\} .
\end{aligned}
$$

Note that $\mathscr{B}^{m, n}$ is totally real except along a $(3 m-2 n-2)$-dimensional plane, where it has CR-singularities of order 1. In [10], Coffman further showed that if $M$ is real-analytic near a nondegenerate CR-singularity, then there is a normalizing transformation that converges, i.e., $M$ is locally biholomorphically equivalent to $\mathscr{B}^{m, n}$ near such a point. We note that the nondegeneracy conditions required for the formal equivalence to hold at a CR-singular point $p \in M$ are full-rank conditions on matrices involving the second-order derivatives of the local graphing functions of $M$ at $p$; see [10, equations (58) and (62) in Section 6]. These conditions will generically yield a codimension 2 set in $M$. When $m=2 k+1$ and $n=3 k, k \geq 2$, the set of CR-singularities is of dimension 1. Thus, by transversality, the CR-singular set of a generic $(2 k+1)$-dimensional $M \subset \mathbb{C}^{3 k}$ consists only of nondegenerate points. Combining this with the density of real-analytic functions (in any fixed $\ell^{\ell}$-norm, $0 \leq \ell<\infty$ ), and Lemma 2.1, we obtain the following crucial preparatory result.

Lemma 2.2. Any smooth embedding $\iota: M \hookrightarrow \mathbb{C}^{3 k}$ of a smooth $(2 k+1)$-dimensional closed manifold admits a small perturbation (in any fixed $\complement^{s}$-norm, $1 \leq s<\infty$ ) that gives a smooth embedding $\iota_{0}: M \hookrightarrow \mathbb{C}^{3 k}$ such that

(i) the set of CR-singularities of $\iota_{0}(M)$ is a finite union of smooth simple closed real curves,

(ii) near any $C R$-singularity, $\iota_{0}(M)$ is locally biholomorphic to the Beloshapka-Coffman normal form $\mathcal{B}^{2 k+1,3 k}$. 
We note that $\mathscr{B}^{2 k+1,3 k}$ is locally polynomially convex at the origin (see Lemma 4.8), which is a property that is invariant under local biholomorphisms. Thus, since a submanifold in $\mathbb{C}^{n}$ is always locally polynomially convex at its totally real points, $\iota_{0}(M)$ in Lemma 2.2 is locally polynomially convex everywhere.

\section{Topological preliminaries}

We collect some algebro-topological results that will play a crucial role in our arguments. Note that the homotopy groups computed in this section are known in the literature, and will be familiar to topologists. However, since our interest lies in finding explicit generators of these groups, we carry out certain computations from scratch.

3.1. Notation. We fix some notation for the rest of this paper. Slightly different coordinates are used for points in even and odd-dimensional spheres, as follows:

(a) For $k \geq 2$,

$$
\begin{aligned}
S^{2 k} & =\left\{(z, w, \zeta, s) \in \mathbb{C} \times \mathbb{C} \times \mathbb{C}^{k-2} \times \mathbb{R}:|z|^{2}+|w|^{2}+\|\zeta\|^{2}+s^{2}=1\right\}, \\
S^{2 k-1} & =\left\{(z, t, \zeta, s) \in \mathbb{C} \times \mathbb{R} \times \mathbb{C}^{k-2} \times \mathbb{R}:|z|^{2}+t^{2}+\|\zeta\|^{2}+s^{2}=1\right\}, \\
D^{2 k} & =\left\{(z, t, \zeta, s) \in \mathbb{C} \times \mathbb{R} \times \mathbb{C}^{k-2} \times \mathbb{R}:|z|^{2}+t^{2}+\|\zeta\|^{2}+s^{2} \leq 1\right\} .
\end{aligned}
$$

(b) In (a) above, when needed, we write $z=x+i y$ and $\zeta=\left(u_{1}+i v_{1}, \ldots, u_{k-2}+i v_{k-2}\right)$.

(c) When convenient, we denote $e^{i \theta} \in S^{1} \subset \mathbb{C}$ by $\theta$, where $\theta \in(-\pi, \pi]$.

We denote the set of all orthonormal $k$-frames in $\mathbb{C}^{n}$ by $W_{k, n}$. An element

$$
A=\left[a_{1}, \ldots, a_{k}\right] \in W_{k, n}
$$

will be represented as an $n \times k$ matrix with orthonormal columns $a_{j}=\left(a_{1 j}, \ldots, a_{n j}\right)^{T} \in \mathbb{C}^{n}$, $j=1, \ldots, k$. When $k=1, W_{1, n}=S^{2 n-1}$, and we switch between the two conventions, with the understanding that

$$
S^{2 n-1} \ni(z, t, \zeta, s) \leftrightarrow\left(\begin{array}{c}
z \\
t+i s \\
\zeta^{T}
\end{array}\right) \in W_{1, n} .
$$

Let $i_{r}: W_{k, n} \mapsto W_{k+r, n+r}$ be the map given by

$$
A=\left(\begin{array}{ccc}
a_{11} & \cdots & a_{1 k} \\
\vdots & \ddots & \vdots \\
a_{n 1} & \cdots & a_{n k}
\end{array}\right) \mapsto\left(\begin{array}{c|c}
A & \mathbf{0}_{n, r} \\
\hline \mathbf{0}_{r, k} & \mathbf{I}_{r, r}
\end{array}\right) .
$$

Note that $i_{r_{1}} \circ i_{r_{2}}=i_{r_{1}+r_{2}}$. We also need to consider $V_{k, n}$, the (noncompact) Stiefel manifold of $\mathbb{C}$-linearly independent $k$-frames in $\mathbb{C}^{n}$. The compact space $W_{k, n}$ is a strong deformation retract of $V_{k, n}$ via Gram-Schmidt orthogonalization. Lastly, $e_{j}$ denotes the vector

$$
(0, \ldots, \underbrace{1}_{z_{j}}, \ldots, 0) \in \mathbb{C}^{n} .
$$


3.2. Higher homotopy groups of complex Stiefel manifolds. To make the exposition as self-contained as possible, we first collect some basic definitions and results. For more details, the reader may consult classical references such as [20] and [1].

A continuous map $p: E \rightarrow B$ between topological spaces is said to be a Hurewicz fibration if it has the homotopy lifting property with respect to all topological spaces, i.e., for any space $Z$, map $g: Z \rightarrow E$ and homotopy $H: Z \times[0,1] \rightarrow B$ such that the following diagram commutes, there is a $G: Z \times[0,1] \rightarrow E$ that extends $g$ and lifts $H$ :

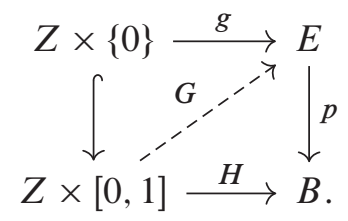

In this case, the fibers or inverse images of points in $B$ are homotopy equivalent (to $F$, say), and the fibration is denoted by

$$
F \hookrightarrow E \stackrel{p}{\rightarrow} B .
$$

Fixing some $b \in B, e \in p^{-1}(b)$ and $f=e$, the fibration induces the following long exact sequence of homotopy groups:

$$
\begin{aligned}
\cdots \longrightarrow \pi_{\ell+1}(B, b) \stackrel{\delta_{\ell}}{\longrightarrow} \pi_{\ell}(F, f) \stackrel{\iota^{*}}{\longrightarrow} \pi_{\ell}(E, e) \\
\stackrel{p^{*}}{\longrightarrow} \pi_{\ell}(B, b) \stackrel{\delta_{\ell-1}}{\longrightarrow} \pi_{\ell-1}(F, f) \longrightarrow \cdots
\end{aligned}
$$

Here, if $H:\left(D^{\ell+1}, \partial D^{\ell+1}\right) \rightarrow(B, b)$ represents an element in $\pi_{\ell+1}(B, b)$, then for any $G: D^{\ell+1} \rightarrow E$ such that the diagram

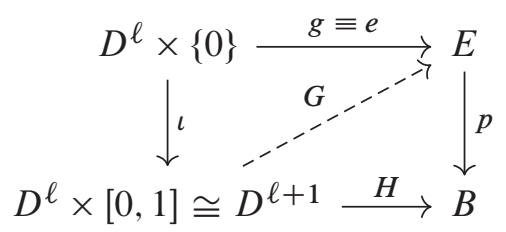

commutes, $\left.G\right|_{\partial D^{\ell+1}}$ induces a well-defined element in $\pi_{\ell}(F, f)$. This is because

$$
G\left(\partial\left(D^{\ell} \times[0,1]\right)\right) \subseteq p^{-1}(b) \cong F
$$

and $G: D^{\ell} \times\{0\} \mapsto e(=f$ in $F)$. The map $\delta_{\ell}$ maps $H$ to this element in $\pi_{\ell}(F, f)$.

Next, we recall the Freudenthal suspension theorem which, in particular, implies the stability of the homotopy groups $\pi_{m+1}\left(S^{m}\right), m \geq 3$. Given a topological space $X$, its suspension is the space

$$
\Sigma X=\left\{(x, \tau) \in X \times[0,1]:\left(x_{1}, \tau\right) \sim\left(x_{2}, \tau\right) \text { when either } \tau=0 \text { or } \tau=1\right\} .
$$

Given a map $f: X \rightarrow Y$, its suspension is the map

$$
\Sigma f: \Sigma X \rightarrow \Sigma Y, \quad(x, s) \mapsto(f(x), s) .
$$

The Freudenthal suspension theorem says that if $X$ is an $n$-connected CW complex, then $f \mapsto \Sigma f$ induces an isomorphism between $\pi_{i}(X)$ and $\pi_{i+1}(\Sigma X)$ when $i<2 n+1$, and an 
epimorphism between $\pi_{2 n+1}(X)$ and $\pi_{2 n+2}(\Sigma X)$. Applying this iteratively to spheres, we obtain that

$$
\pi_{2 k}\left(S^{2 k-1}\right) \cong \pi_{4}\left(S^{3}\right) \cong \mathbb{Z}_{2}, \quad k \geq 2, \quad \mathbb{Z} \cong \pi_{3}\left(S^{2}\right) \rightarrow \pi_{4}\left(S^{3}\right) .
$$

Furthermore, since the Hopf fibration given by $\mathfrak{h}:(z, w) \mapsto\left(2 \bar{z} w,|z|^{2}-|w|^{2}\right)$ is a generator of $\pi_{3}\left(S^{2}\right)$, we obtain the following generator of $\pi_{2 k}\left(S^{2 k-1}\right)$ via suspensions:

(3.2) $\mathfrak{h}_{k}:(z, w, \zeta, s) \mapsto\left(2 \bar{z} w,|z|^{2}-|w|^{2}, \zeta \sqrt{1+|z|^{2}+|w|^{2}}, s \sqrt{1+|z|^{2}+|w|^{2}}\right)$.

In [17] (results announced in [16]), fibrations and suspensions are used to compute certain higher homotopy groups of complex Stiefel manifolds. In particular, it is shown that

$$
\pi_{\ell}\left(W_{k, n}\right)= \begin{cases}0 & \text { if } \ell \leq 2(n-k), \\ \mathbb{Z} & \text { if } \ell=2(n-k)+1, \\ 0 & \text { if } \ell=2(n-k)+2, n-k \text { is odd } \\ \mathbb{Z}_{2} & \text { if } \ell=2(n-k)+2, n-k \text { is even }\end{cases}
$$

We retrace this technique to compute explicit generators of $\pi_{2 k-1}\left(W_{2 k+1,3 k}\right), \pi_{2 k}\left(W_{2 k+1,3 k}\right)$, $k \geq 2$.

Lemma 3.1. For any $k \geq 2$, the map

$$
\alpha: S^{2 k-1} \ni(z, t, \zeta, s) \mapsto i_{2 k}(z, t, \zeta, s)=\left(\begin{array}{c|c}
z & \\
t+i s & \mathbf{0}_{k, 2 k} \\
\zeta^{T} & \\
\hline \mathbf{0}_{2 k, 1} & \mathbf{I}_{2 k, 2 k}
\end{array}\right)
$$

represents a generator of $\pi_{2 k-1}\left(W_{2 k+1,3 k}\right) \cong \mathbb{Z}$.

When $k>2$ is odd, the map

$$
\beta: S^{2 k} \ni(z, w, \zeta, s) \mapsto\left(\begin{array}{c|c}
2 \bar{z} w & \\
1-2|w|^{2}+i 2|w| s & \mathbf{0}_{k, 2 k} \\
2|w| \zeta^{T} & \\
\hline \mathbf{0}_{2 k, 1} & \mathbf{I}_{2 k, 2 k}
\end{array}\right)
$$

represents a generator of $\pi_{2 k}\left(W_{2 k+1,3 k}\right) \cong \mathbb{Z}_{2}$.

Proof. To prove the lemma, we show that the generators of $\pi_{\ell}\left(W_{2 k+1,3 k}\right)$ essentially descend to generators of $\pi_{\ell}\left(S^{2 k-1}\right), \ell=2 k-1,2 k$.

We break the proof into three steps ((A), (B) and (C) below). First, for $1 \leq k \leq n$, we consider the fibration

$$
W_{k-1, n-1} \stackrel{i_{1}}{\rightarrow} W_{k, n} \stackrel{p}{\rightarrow} S^{2 n-1},
$$

where $p:\left[v_{1}, \ldots, v_{k}\right] \mapsto v_{k}$. Ignoring the basepoints, we obtain the following long exact sequence:

$$
\cdots \longrightarrow \pi_{\ell+1}\left(S^{2 n-1}\right) \stackrel{\delta_{\ell}}{\longrightarrow} \pi_{\ell}\left(W_{k-1, n-1}\right) \stackrel{i_{1}^{*}}{\longrightarrow} \pi_{\ell}\left(W_{k, n}\right) \stackrel{p^{*}}{\longrightarrow} \pi_{\ell}\left(S^{2 n-1}\right) \longrightarrow \cdots,
$$


which, applied iteratively, proves that for $\ell<2 n-2 m, \pi_{\ell}\left(W_{k-m, n-m}\right) \cong \pi_{\ell}\left(W_{k, n}\right)$ via $i_{m}^{*}$. In particular,

(A) for $k \geq 1, \pi_{2 k}\left(W_{2 k+1,3 k}\right) \cong \pi_{2 k}\left(W_{2, k+1}\right)$ via $i_{2 k-1}^{*}$.

and, $\pi_{2 k-1}\left(S^{2 k-1}\right) \cong \pi_{2 k-1}\left(W_{2 k+1,3 k}\right)$ via the isomorphism $i_{2 k}^{*}$. Note that the latter fact proves the first half of our claim.

Next, we consider a particular case of (3.4),

$$
S^{2 k-1} \cong W_{1, k} \stackrel{i_{1}}{\longrightarrow} W_{2, k+1} \stackrel{p}{\longrightarrow} S^{2 k+1},
$$

to obtain the long exact sequence

$$
\begin{gathered}
\cdots \longrightarrow \pi_{2 k+1}\left(S^{2 k+1}\right) \stackrel{\delta_{2 k}}{\longrightarrow} \pi_{2 k}\left(S^{2 k-1}\right) \stackrel{i_{1}^{*}}{\longrightarrow} \pi_{2 k}\left(W_{2, k+1}\right) \\
\stackrel{p^{*}}{\longrightarrow} \underbrace{\pi_{2 k}\left(S^{2 k+1}\right)}_{=0} \longrightarrow \cdots .
\end{gathered}
$$

In order to understand the map $\delta_{2 k}$, we fix $b=e_{k+1} \in \mathbb{C}^{k+1}$ and $e=\left[e_{k}, e_{k+1}\right] \in W_{2, k+1}$, and consider the following specific case of diagram (3.1):

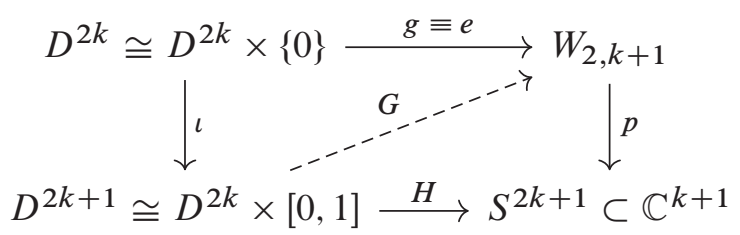

for any $H=\left(h_{1}, \ldots, h_{k+1}\right):\left(D^{2 k+1}, \partial D^{2 k+1}\right) \rightarrow\left(S^{2 k+1}, b\right)$. When $k+1$ is even, the above diagram commutes if $G:\left(D^{2 k+1}, \partial D^{2 k+1}\right) \rightarrow\left(W_{2, k+1}, e\right)$ is set as

$$
\left(D^{2 k+1}\right) \ni Z \mapsto\left(\begin{array}{cc}
h_{2}(Z) & h_{1}(Z) \\
-h_{1}(Z) & h_{2}(Z) \\
\vdots & \vdots \\
h_{k+1}(Z) & h_{k}(Z) \\
-h_{k}(Z) & h_{k+1}(Z)
\end{array}\right) .
$$

In particular,

$$
\left.G\right|_{\partial D^{2 k+1}} \equiv\left[e_{k}, e_{k+1}\right] \subset p^{-1}\left(e_{k+1}\right) \cong S^{2 k-1},
$$

which induces the trivial element in $\pi_{2 k}\left(S^{2 k-1}\right)$. Thus,

(B) when $k$ is odd, $\delta_{2 k}=0$ in (3.5) and, hence, $\pi_{2 k}\left(S^{2 k-1}\right) \cong \pi_{2 k}\left(W_{2 k+1,3 k}\right)$ via $i_{1}^{*}$.

Combining (A) and (A), we have that $\pi_{2 k}\left(W_{2 k+1,3 k}\right) \cong \pi_{2 k}\left(S^{2 k-1}\right) \cong \mathbb{Z}_{2}$ via $i_{2 k}^{*}$. Moreover, owing to (3.2), $\psi=i_{2 k} \circ \mathfrak{h}_{k}$ is a generator of $\pi_{2 k}\left(W_{2 k+1,3 k}\right)$. To complete our proof, we show that

(C) $\psi$ and $\beta$ are homotopic as maps from $S^{2 k}$ into $W_{2 k+1,3 k}$.

Note that we may write $\beta=i_{2 k} \circ h$, where $h: S^{2 k} \rightarrow S^{2 k-1}$ is given by

$$
h:(z, w, \zeta, s) \mapsto\left(2 \bar{z} w, 1-2|w|^{2}, 2|w| \zeta, 2|w| s\right) .
$$


Then, the map $H: S^{2 k} \times[0,1] \rightarrow W_{2 k+1,3 k}$ which sends $(z, w, \zeta, s, \tau)$ to

$$
\begin{aligned}
& i_{2 k}\left(2 \bar{z} w,|z|^{2}-|w|^{2}+\tau\|\zeta\|^{2}+\tau s^{2},(\zeta, s)\right. \\
& \left.\quad \sqrt{2(1-\tau)|z|^{2}+2(1+\tau)|w|^{2}+\left(1-\tau^{2}\right)\left(\|\zeta\|^{2}+s^{2}\right)}\right)
\end{aligned}
$$

is a homotopy between $\psi$ and $\beta$. This completes the proof of our lemma.

Remark 3.2. Viewing $S^{2 k-1}$ as a sphere in $\mathbb{C}^{k}$ with complex orientation and coordinates $(z, t+i s, \zeta)$, we fix the convention that $[\alpha]=1$ in $\mathbb{Z}$. Now, for any continuous map

$$
f: S^{2 k-1} \mapsto \pi_{2 k-1}\left(W_{2 k+1,3 k}\right) \cong \mathbb{Z},
$$

the homotopy class of $f$ is a well-defined integer, which we denote by $\operatorname{deg}(f)$ and call the degree of $f$. The proof of Lemma 3.1 shows that if $g$ is a continuous self-map of $S^{2 k-1}$, then

$$
\operatorname{deg}(f \circ g)=\operatorname{deg}(f) \operatorname{deg}_{S^{2 k-1}}(g)
$$

where $\operatorname{deg}_{S^{2 k-1}}(\cdot)$ is to be understood as the degree of a continuous self-map of $S^{2 k-1}$.

3.3. Maps from $S^{1} \times S^{2 k-1}$ into certain Stiefel manifolds. In this subsection, we denote by $[X, Y]$ the set of homotopy classes of continuous maps from $X$ to $Y$, where $X$ and $Y$ are topological spaces. As a consequence of the Freudenthal suspension theorem, it is known that the set $[X, Y]$ is canonically an abelian group if $Y$ is $n$ connected and $X$ is a CW complex of dimension at most $2 n$; see [24, Corollary 3.2.3]. This is indeed the case when $X=S^{1} \times S^{2 k-1}$ and $Y=W_{2 k+1,3 k}, k \geq 2$. Thus, $\left[S^{1} \times S^{2 k-1}, W_{2 k+1,3 k}\right]$ is an abelian group. In fact, we can be more precise.

Lemma 3.3. Let $k \geq 2$. Then

$$
\begin{aligned}
{\left[S^{1} \times S^{2 k-1}, W_{2 k+1,3 k}\right] } & \cong \pi_{2 k-1}\left(W_{2 k+1,3 k}\right) \oplus \pi_{2 k}\left(W_{2 k+1,3 k}\right) \\
& \cong \begin{cases}\mathbb{Z} & \text { when } k \text { is even, } \\
\mathbb{Z} \oplus \mathbb{Z}_{2} & \text { when } k \text { is odd } .\end{cases}
\end{aligned}
$$

In the first case, the isomorphism is induced by the map $f \mapsto f_{\text {slice }}:=\left.f\right|_{\{\cdot\} \times S^{2 k-1}}$. In the second case, $f \mapsto f_{\text {slice }}$ determines the projection onto the first factor.

Proof. In order to describe the abelian group $\left[S^{1} \times S^{2 k-1}, W_{2 k+1,3 k}\right]$, we consider the following cofiber sequence (see [4, Chapter 3]):

$$
S^{2 k-1} \stackrel{\mu}{\longrightarrow} S^{1} \vee S^{2 k-1} \stackrel{\iota}{\longrightarrow} S^{1} \times S^{2 k-1} \stackrel{\gamma}{\longrightarrow} S^{2 k} \stackrel{\Sigma \mu}{\longrightarrow} S^{2} \vee S^{2 k} \stackrel{\Sigma \iota}{\longrightarrow} \cdots,
$$

where

- $\mu$ is the attaching map of the top cell of $S^{1} \times S^{2 k-1}$,

- $\iota$ is the cellular inclusion, and

- $\gamma$ is the quotient map $S^{1} \times S^{2 k-1} \rightarrow S^{1} \times S^{2 k-1} / S^{1} \vee S^{2 k-1} \cong S^{2 k}$. 
For any CW complex $X$, (3.6) induces the following long exact sequence of pointed sets (on fixing basepoints).

$$
1 \longrightarrow\left[S^{2 k}, X\right] \stackrel{\gamma_{*}}{\longrightarrow}\left[S^{1} \times S^{2 k-1}, X\right] \stackrel{i_{*}}{\longrightarrow}\left[S^{1} \vee S^{2 k-1}, X\right] \stackrel{\mu_{*}}{\longrightarrow}\left[S^{2 k-1}, X\right] .
$$

Here, we have used the fact that the suspension of an attaching map is nullhomotopic. Further, $\mu_{*}: \pi_{1}(X) \times \pi_{2 k-1}(X) \rightarrow \pi_{2 k-1}(X)$ is the Whitehead product, which vanishes if $\pi_{1}(X)=0$. Thus, for $X=W_{2 k+1,3 k}, k \geq 2$, we get the following exact sequence of abelian groups:

$$
0 \rightarrow \pi_{2 k}\left(W_{2 k+1,3 k}\right) \rightarrow\left[S^{1} \times S^{2 k-1}, W_{2 k+1,3 k}\right] \rightarrow \pi_{2 k-1}\left(W_{2 k+1,3 k}\right) \rightarrow 0 .
$$

Based on (3.3), we have the result.

Next, we describe the generators of these groups. In particular, when $k$ is odd, we obtain two nonhomotopic maps from $S^{1} \times S^{2 k-1}$ into $W_{2 k+1,3 k}$ that restrict to homotopic maps on each slice $\{\cdot\} \times S^{2 k-1}$.

Lemma 3.4. Let $k \geq 2$. Consider the following maps from $S^{1} \times S^{2 k-1}$ into $W_{2 k+1,3 k}$ :

$$
\begin{aligned}
& f_{1}:(\theta, z, t, \zeta, s) \mapsto i_{2 k}(z, t, \zeta, s), \\
& f_{2}:(\theta, z, t, \zeta, s) \mapsto i_{2 k}\left(z e^{i \theta}, t, \zeta, s\right) .
\end{aligned}
$$

Then $\left[\left(f_{1}\right)_{\text {slice }}\right]=\left[\left(f_{2}\right)_{\text {slice }}\right]=+1$ which, for even $k$, implies that $\left[f_{1}\right]=\left[f_{2}\right]$. However, one has $\left[f_{1}\right] \neq\left[f_{2}\right]$, when $k$ is odd.

Proof. We note that owing to Lemma 3.3 and Lemma 3.1, we only need to show that $\left[f_{1}\right] \neq\left[f_{2}\right]$ when $k$ is odd. We fix the basepoint $b=(0,1,0, \ldots, 0) \in S^{2 k-1} ;$ let $S^{1} \times_{b} S^{2 k-1}$ denote the pinched torus $S^{1} \times S^{2 k-1} / S^{1} \times\{b\}$, where we use the same coordinates as those on $S^{1} \times S^{2 k-1}$, but denote the pinched point by $[\theta, b]$. Since $f_{j}(\theta, b)=i_{2 k}(b)$ for all $\theta \in[0,2 \pi]$, $j=1,2$, these maps factor through $S^{1} \times_{b} S^{2 k-1}$ (via $f_{j}^{*}$, say):

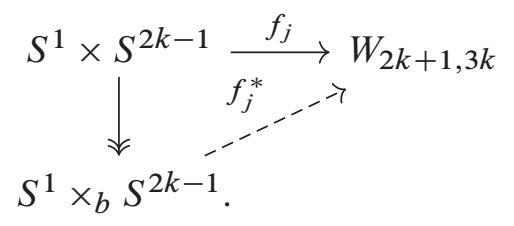

Furthermore, any homotopy $H: S^{1} \times S^{2 k-1} \times[0,1] \rightarrow W_{2 k+1,3 k}$ between $f_{1}$ and $f_{2}$ descends to a homotopy between $f_{1}^{*}$ and $f_{2}^{*}$ as long as the 2-sphere given by $H\left(S^{1} \times\{b\} \times[0,1]\right)$ in $W_{2 k+1,3 k}$ is nullhomotopic. This is always the case since $\pi_{2}\left(W_{2 k+1,3 k}\right)=0$. Thus, it suffices to show that $f_{1}^{*}$ and $f_{2}^{*}$ are nonhomotopic as maps from $S^{1} \times_{b} S^{2 k-1}$ into $W_{2 k+1,3 k}$. by

For this, we consider the maps $g_{j}:=f_{j}^{*} \circ g$, where $g: S^{2 k} \rightarrow S^{1} \times_{b} S^{2 k-1}$ is given

$$
(z, w, \zeta, s) \mapsto \begin{cases}\left(\arg w, 2 \bar{z}|w|, 1-2|w|^{2}, 2|w| \zeta, 2|w| s\right), & w \neq 0, \\ {[\theta, b],} & w=0 .\end{cases}
$$

Any homotopy between $f_{1}^{*}$ and $f_{2}^{*}$ will extend to a homotopy between $g_{1}$ and $g_{2}$. Since $g_{2}=\beta$ is a generator of $\pi_{2 k}\left(W_{2 k+1,3 k}\right)=\mathbb{Z}_{2}$ (as shown in Lemma 3.1), our proof is complete 

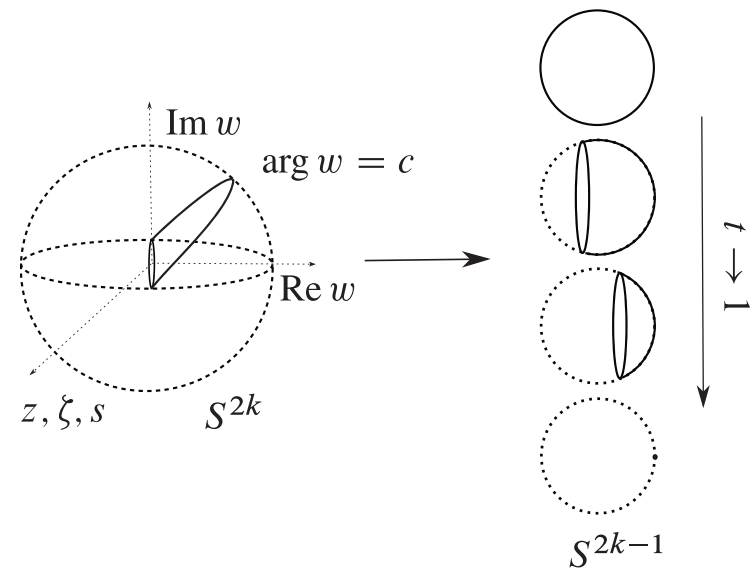

Figure 1. Evolution of the homotopy $H$ along any slice $\arg w=c$.

if we show that $g_{1}$ is nullhomotopic. For this, it suffices to show that $\widetilde{g}: S^{2 k} \rightarrow S^{2 k-1}$ given by

$$
\widetilde{g}:(z, w, \zeta, s) \mapsto\left(2 \bar{z}|w|, 1-2|w|^{2}, 2|w| \zeta, 2|w| s\right)
$$

is nullhomotopic, for $g_{2}=i_{2 k} \circ \widetilde{g}$. The homotopy

$$
H: S^{2 k} \times[0,1] \rightarrow S^{2 k-1}
$$

given by

$$
H:((z, w, \zeta, s), \tau) \mapsto h(\eta(\tau,|w|) z, \tau+(1-\tau)|w|, \eta(\tau,|w|) \zeta, \eta(\tau,|w|) s),
$$

where

$$
\eta(\tau, r)=\frac{\sqrt{1-(\tau+(1-\tau) r)^{2}}}{\sqrt{1-r^{2}}},
$$

resolves this matter; see the schematic in Figure 1. The map $H$ is well-defined when $|w|=1$ because

$$
\lim _{r \rightarrow 1^{-}} \eta(\tau, r)=1-\tau \quad \text { for all } \tau \in[0,1]
$$

Moreover,

$$
H((z, w, \zeta, s), 0)=h(z,|w|, \zeta, s)=h(z, w, \zeta, s)
$$

and

$$
H((z, w, \zeta, s), 1) \equiv(0,-1,0,0)
$$

This concludes our proof.

\section{Tubular neighborhoods of curves of CR-singularities}

We now focus on neighborhoods of certain simple closed curves in $(2 k+1)$-dimensional submanifolds of $\mathbb{C}^{3 k}$. We introduce a specialized notion of cobordism between the boundaries of these neighborhoods, and produce polynomially convex models to represent all the possible classes of this topological equivalence. This section encapsulates the main technical part of our construction. 
4.1. The index of a parametrized tube enclosing curve. The precise class of neighborhoods under consideration is as follows.

Definition 4.1. Suppose $\mathcal{N} \subset \mathbb{C}^{3 k}$ is a compact submanifold with boundary that admits a smooth parametrization $F: S^{1} \times D^{2 k} \rightarrow \mathcal{N}$ such that

(a) $F$ is totally real on $\left(S^{1} \times D^{2 k}\right) \backslash\left(S^{1} \times\{0\}\right)$,

(b) $\operatorname{dim}_{\mathbb{C}} T_{p}^{c} \mathcal{N}$ is constant over all $p \in F\left(S^{1} \times\{0\}\right)$.

Then we call $\mathcal{N}$ a tube enclosing a curve in $\mathbb{C}^{3 k}$, or a tube enclosing a curve, since the ambient dimension is fixed. We call $F$ an admissible parametrization of $\mathcal{N}$. We single out two special cases.

(i) If $\mathcal{N}$ is totally real everywhere, we call it a fully-TR tube.

(ii) If the CR dimension of $\mathcal{N}$ is positive on $\gamma:=F\left(S^{1} \times\{0\}\right)$, then $\gamma$ is independent of $F$. In this case, we say that $\mathcal{N}$ is a tube enclosing the $C R$-singular curve $\gamma$, or simply a tube enclosing a CR-singular curve.

In all the cases above, the word parametrized is appended to the terminology when discussing the pair $(\mathcal{N}, F)$.

As before, we use $(\theta, z=x+i y, t, \zeta, s)$ for points in $S^{1} \times D^{2 k}$. Further, we fix the frames

$$
\vartheta=\frac{\partial}{\partial \theta} \quad \text { and } \quad \sigma=\left(\vartheta, \sigma^{\prime}\right)
$$

for the tangent bundles $T\left(S^{1} \times\{0\}\right)$ and $T\left(S^{1} \times D^{2 k}\right)$, respectively, and

$$
\sigma^{\prime}=\left(\frac{\partial}{\partial x}, \frac{\partial}{\partial y}, \frac{\partial}{\partial t}, \frac{\partial}{\partial s}, \frac{\partial}{\partial u_{1}}, \frac{\partial}{\partial v_{1}}, \ldots, \frac{\partial}{\partial u_{k-2}}, \frac{\partial}{\partial v_{k-2}}\right)
$$

for the normal bundle $N\left(S^{1} \times\{0\}\right)$ of $S^{1} \times\{0\}$ in $S^{1} \times D^{2 k}$. Given a diffeomorphism

$$
F: S^{1} \times D^{2 k} \rightarrow \mathbb{C}^{3 k}
$$

that is totally real away from the curve $F\left(S^{1} \times\{0\}\right)$, and $p \in S^{1}$, we define the maps

$$
\widehat{F}: S^{1} \times S^{2 k-1} \rightarrow W_{2 k+1,3 k}, \quad \xi \mapsto\left[\left(F_{*} \sigma\right)(F(\xi))\right]^{\mathrm{GS}}=[D F(\xi)]^{\mathrm{GS}},
$$

and

$$
\widehat{F}_{p \text {-slice }}: S^{2 k-1} \rightarrow W_{2 k+1,3 k}, \quad \eta \mapsto\left[\left(F_{*} \sigma\right)(F(p, \eta))\right]^{\mathrm{GS}}=[D F(p, \eta)]^{\mathrm{GS}},
$$

where $\left[\left(F_{*} \sigma\right)(F(\cdot))\right]^{\mathrm{GS}}$ is the Gram-Schmidt orthogonalization of the frame $\left(F_{*} \sigma\right)(F(\cdot))$, viewed as a full-rank $(3 k) \times(2 k+1)$ complex matrix. The map $\widehat{F}$ will be referred to as the frame map of the parametrized tube $\left(\mathcal{N}=F\left(S^{1} \times D^{2 k}\right), F\right)$.

Note that $\widehat{F}, \widehat{F}_{p \text {-slice }}$ induce elements in $\left[S^{1} \times S^{2 k-1}, W_{2 k+1,3 k}\right]$ and $\pi_{2 k-1}\left(W_{2 k+1,3 k}\right)$, respectively. Since $W_{2 k+1,3 k}$ is a strong deformation retract of $V_{2 k+1,3 k}$, we will often abuse notation and drop the notation $[\cdot]^{\mathrm{GS}}$ to view $\widehat{F}$ and $\widehat{F}_{p \text {-slice }}$ as maps into $V_{2 k+1,3 k}$.

In this subsection, we focus on the homotopy class induced by $\widehat{F}_{p \text {-slice. }}$. It is clear that this

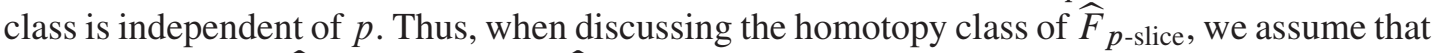
$p=1$ and denote $\widehat{F}_{p \text {-slice }}$ simply by $\widehat{F}_{\text {slice }}$. Furthermore, in view of Remark 3.2, the following definition is well-defined. 
Definition 4.2. The index of a parametrized tube $(\mathcal{N}, F)$ enclosing a curve is defined as

$$
\operatorname{ind}_{F}(\mathcal{N})=\operatorname{deg}\left(\widehat{F}_{p \text {-slice }}\right) \quad \text { for any } p \in S^{1} \text {. }
$$

To understand the dependence of this index on $F$, we note that $\sigma^{\prime}$ assigns an orientation, say $\mathfrak{D}$, on the normal bundle of $S^{1} \times\{0\}$ in $S^{1} \times D^{2 k}$. Given a self-diffeomorphism, $\varphi$, of $S^{1} \times D^{2 k}$ that maps $S^{1} \times\{0\}$ to itself, if $\varphi_{*} \sigma^{\prime}$ induces the same orientation $\mathfrak{D}$ on $N\left(S^{1} \times\{0\}\right)$, we say that $\varphi$ is fiberwise orientation preserving. Otherwise, $\varphi$ is called fiberwise orientation reversing. We now show that, modulo its sign, $\operatorname{ind}_{F}(\mathcal{N})$ is independent of $F$.

Lemma 4.3. Let $F$ and $G$ be admissible parametrizations of $\mathcal{N}$, a tube enclosing a curve in $\mathbb{C}^{3 k}$. Then

$$
\operatorname{ind}_{G}(\mathcal{N})= \begin{cases}\operatorname{ind}_{F}(\mathcal{N}) & \text { if } F^{-1} \circ G \text { is fiberwise orientation-preserving, } \\ -\operatorname{ind}_{F}(\mathcal{N}) & \text { if } F^{-1} \circ G \text { is fiberwise orientation-reversing. }\end{cases}
$$

Moreover, if $U$ is a neighborhood of $F\left(A \times S^{2 k}\right)$, where $A \subset S^{1}$ is an arc containing $p=1$, and $H: U \rightarrow \mathbb{C}^{3 k}$ is a biholomorphism, then

$$
\operatorname{ind}_{F}(\mathcal{N})=\operatorname{deg}\left(\widehat{(H \circ F}_{\text {slice }}\right) .
$$

Proof. Let $\varphi:=F^{-1} \circ G$. Since $\sigma$ is the coordinate frame on $S^{1} \times D^{2 k}$, we may write

$$
\widehat{G}_{\text {slice }}(\eta)=\left(G_{*} \sigma\right)(G(1, \eta))=D G(1, \eta)=D F(\varphi(1, \eta)) \cdot D \varphi(1, \eta), \quad \eta \in S^{2 k-1} .
$$

Since det $D \varphi \neq 0$ on $S^{1} \times D^{2 k}$, it follows that the map $\left.D \varphi(1, \cdot)\right|_{S^{2 k-1}}$, extends to a map from $D^{2 k}$ into $\mathrm{GL}_{\mathbb{C}}(2 k+1)$, and is, thus, homotopic to the constant identity map in $\mathrm{GL}_{\mathbb{C}}(2 k+1)$. As $V_{2 k+1,3 k}$ is closed under the action of $\mathrm{GL}_{\mathbb{C}}(2 k+1)$ via multiplication from the right, $\widehat{G}_{\text {slice }}$ is homotopic to the map

$$
\eta \mapsto D F(\varphi(1, \eta)), \quad \eta \in S^{2 k-1} .
$$

Next, letting $\eta^{*}=(\bar{z}, t, \zeta, s)$, for $\eta=(z, t, \zeta, s) \in D^{2 k}$, we observe that $\left.\varphi\right|_{\{1\} \times S^{2 k-1}}$ is homotopic to the identity map $\mathrm{i}$ on $S^{2 k-1}$ when $\varphi$ is fiberwise orientation-preserving, and to $\mathrm{i}^{*}: \eta \mapsto \eta^{*}, \eta \in S^{2 k-1}$, when $\varphi$ is fiberwise orientation-reversing. Thus, $\widehat{G}_{\text {slice }}$ is homotopic in $V_{2 k+1,3 k}$ to

$$
\begin{cases}D F(\mathrm{i}(1, \cdot)) & \text { if } F^{-1} \circ G \text { is fiberwise orientation-preserving }, \\ D F\left(\mathrm{i}^{*}(1, \cdot)\right) & \text { if } F^{-1} \circ G \text { is fiberwise orientation-reversing. }\end{cases}
$$

Since $\operatorname{deg}_{S^{2 k-1}}(\mathrm{i})=+1$ and $\operatorname{deg}_{S^{2 k-1}}\left(\mathrm{i}^{*}\right)=-1$, the first part of our claim now follows from Remark 3.2.

For the second part of our claim, note that

$$
\widehat{(H \circ F)}_{\text {slice }}(\eta)=D_{\mathbb{C}} H(F(1, \eta)) \cdot D F(1, \eta), \quad \eta \in S^{2 k-1} .
$$

But, $\left.\left(\left(D_{\mathbb{C}} H\right) \circ F\right)(1, \cdot)\right|_{S^{2 k-1}}$ extends to a map from $D^{2 k}$ into $\mathrm{GL}_{\mathbb{C}}(3 k)$. Thus, it is homotopic to the constant identity map in $\mathrm{GL}_{\mathbb{C}}(3 k)$. Now, since, $V_{2 k+1,3 k}$ is closed under the action of $\mathrm{GL}_{\mathbb{C}}(3 k)$ via multiplication from the left, we are done. 
Remark 4.4. From the lemma above, it is clear that

$$
\operatorname{ind}_{F \circ \varphi}(\mathcal{N})=-\operatorname{ind}_{F}(\mathcal{N}),
$$

where $\varphi: S^{1} \times D^{2 k} \rightarrow S^{1} \times D^{2 k}$ is given by $(\theta, \eta) \mapsto\left(g(\theta), \eta^{*}\right)$, for some diffeomorphism $g: S^{1} \rightarrow S^{1}$.

4.2. Totally real cylindrical cobordisms between tubes enclosing curves. We now discuss a notion of equivalence between parametrized tubes enclosing curves, under which one tube can be essentially replaced by another without any addition of CR-singularities. We first fix some additional notation.

(a) Let $\mathbf{T}=[0,1] \times S^{1} \times S^{2 k-1}$.

(b) For $\delta \in\left(0, \frac{1}{2}\right)$, let $\mathbf{T}_{\delta}=[0, \delta] \times S^{1} \times S^{2 k-1}$ and $\mathbf{T}_{1-\delta}=[1-\delta, 1] \times S^{1} \times S^{2 k-1}$.

(c) Let $L_{c}:[c, c+1) \times S^{1} \times S^{2 k-1} \rightarrow S^{1} \times\left(D^{2 k} \backslash\{0\}\right)$ be the map given by

$$
(\tau, \theta, \eta) \mapsto(\theta,(c+1-\tau) \eta)
$$

Definition 4.5. Suppose $\left(\mathcal{N}_{1}, F_{1}\right)$ and $\left(\mathcal{N}_{2}, F_{2}\right)$ are parametrized tubes enclosing curves in $\mathbb{C}^{3 k}$. If they are disjoint, they are said to be totally real cylindrically cobordant or TRC cobordant if there exists a totally real embedding $F: \mathbf{T} \rightarrow \mathbb{C}^{3 k}$ such that, for some sufficiently small $\delta$,

$$
F(\tau, \theta, \eta)= \begin{cases}\left(F_{1} \circ L_{0}\right)(\tau, \theta, \eta) & \text { on } \mathbf{T}_{\delta}, \\ \left(F_{2} \circ L_{1-\delta}\right)(\tau, \theta, \eta) & \text { on } \mathbf{T}_{1-\delta},\end{cases}
$$

for $(\tau, \theta, \eta) \in \mathbf{T}$ (see Figure 2). If they are not disjoint, they are said to be totally real cylindrically cobordant if there is some translation $\tau$ of $\mathbb{C}^{3 k}$ such that $\tau\left(\mathcal{N}_{2}\right) \cap \mathcal{N}_{1}=\emptyset$ and $\left(\mathcal{N}_{1}, F_{2}\right)$ and $\left(\tau\left(\mathcal{N}_{2}\right), F_{2} \circ \tau\right)$ are totally real cylindrically cobordant in the sense discussed above.

Before we give a characterization of TRC cobordant pairs, we state a geometric consequence of (4.2). This result will be referred to as the neighborhood replacement result in this paper.

Proposition 4.6. Suppose $\left(\mathcal{N}_{1}, F_{1}\right)$ and $\left(\mathcal{N}_{2}, F_{2}\right)$ are disjoint totally-real cobordant parametrized tubes enclosing curves in $\mathbb{C}^{3 k}$. Then, for a sufficiently small $\varepsilon>0$, there is a smooth embedding $\Psi_{\mathcal{N}_{1}, \mathcal{N}_{2}}: S^{1} \times 2 D^{2 k} \rightarrow \mathbb{C}^{3 k}$ such that

(1) $\Psi_{\mathcal{N}_{1}, \mathcal{N}_{2}}$ is totally real on $\left(S^{1} \times 2 D^{2 k}\right) \backslash\left(S^{1} \times\{0\}\right)$,

(2) $\Psi_{\mathcal{N}_{1}, \mathcal{N}_{2}}(\theta, r \eta)=F_{1}(\theta,(r-1) \eta)$ for $r \in[2-\varepsilon, 2]$ and $(\theta, \eta) \in S^{1} \times S^{2 k-1}$,

(3) $\Psi_{\mathcal{N}_{1}, \mathcal{N}_{2}}$ coincides with $F_{2}$ on $S^{1} \times D^{2 k}$.

Proof. Let $F: \mathbf{T} \rightarrow \mathbb{C}^{3 k}$ be an embedding satisfying (4.2) and $\tau:[1-\delta, 2-\delta] \rightarrow[0,1]$ a smooth bijective map such that

$$
\tau(r)= \begin{cases}2-\delta-r & \text { when } r \in[1-\delta, 1], \\ 2-r & \text { when } r \in[2-\delta, 2] .\end{cases}
$$




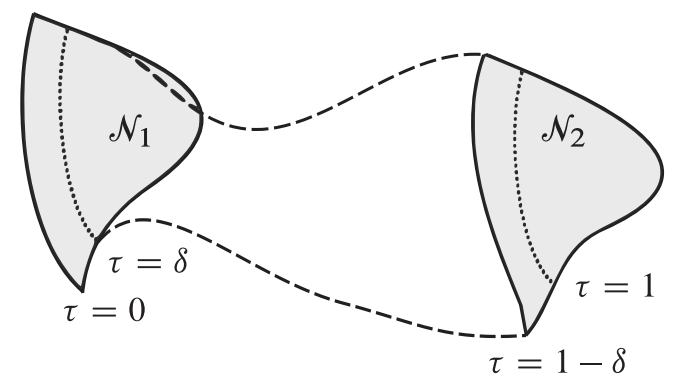

Figure 2. $\quad \mathcal{N}_{1} \cup F(\mathbf{T}) \cup \mathcal{N}_{2}$.

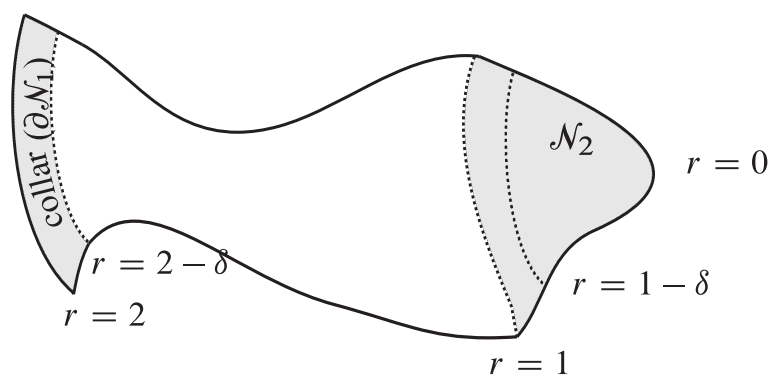

Figure 3. $\quad \Xi\left(S^{1} \times 2 D^{2 k}\right)=F(\mathbf{T}) \cup \mathcal{N}_{2}$.

Then the map $\Xi: S^{1} \times 2 D^{2 k}$ given by

$$
\Xi(\theta, r \eta)= \begin{cases}F_{2}(\theta, r \eta) & \text { when } r \in[0,1-\delta], \\ F_{2}(\theta, r \eta)=F(2-\delta-r, \theta, \eta) & \text { when } r \in[1-\delta, 1], \\ F(\tau(r), \theta, \eta) & \text { when } r \in[1,2-\delta], \\ F_{1}(\theta,(r-1) \eta)=F(2-r, \theta, \eta) & \text { when } r \in[2-\delta, 2],\end{cases}
$$

is smooth (see Figure 3). Furthermore, $\Xi$ is a local embedding everywhere in the interior of its domain, and is an embedding in an open neighborhood of $K=\left(S^{1} \times D^{2 k}\right) \cup \partial\left(S^{1} \times 2 D^{2 k}\right)$. Thus, by the relative version of the weak Whitney embedding theorem, there is an embedding $\Psi_{\mathcal{N}_{1}, \mathcal{N}_{2}}$ with the desired properties.

We now state the aforementioned algebro-topological characterization of pairs of TRC cobordant parametrized tubes enclosing curves. In particular, we see that the index is only a partial invariant in this respect.

Lemma 4.7. Suppose $\left(\mathcal{N}_{1}, F_{1}\right)$ and $\left(\mathcal{N}_{2}, F_{2}\right)$ are disjoint parametrized tubes enclosing curves in $\mathbb{C}^{3 k}$. Then they are TRC cobordant if and only if their frame maps, $\widehat{F}_{1}$ and $\widehat{F}_{2}$, induce the same element in $\left[S^{1} \times S^{2 k-1}, W_{2 k+1,3 k}\right]$.

Proof. First, we fix a smooth translation-invariant (in $\tau$ ) field of frames on $\mathbf{T}$ as follows:

$$
\sigma_{\mathbf{T}}(\tau, \theta, \eta)=\left(L_{\tau}^{*} \sigma\right)(\tau, \theta, \eta) .
$$

For any parametrization $g: S^{1} \times D^{2 k} \rightarrow \mathbb{C}^{3 k}$, let $g^{c}=g \circ L_{c}$. Because of the translationinvariance,

$$
\left(g_{*}^{c} \sigma_{\mathbf{T}}\right)\left(g^{c}(c, \cdot)\right)=\left.\left(g_{*} \sigma\right)(g(\cdot))\right|_{S^{1} \times S^{2 k-1}},
$$


and, if $\tau>c$, then $\left(g_{*}^{c} \sigma_{\mathbf{T}}\right)\left(g_{c}(\tau, \cdot)\right)$ is homotopic to $\left.\left(g_{*} \sigma\right)(g(\cdot))\right|_{S^{1} \times S^{2 k-1}}$. In short, setting

$$
\widehat{g}_{\tau}^{c}: \xi \mapsto\left[\left(g_{*}^{c} \sigma_{\mathbf{T}}\right)\left(g^{c}(\tau, \xi)\right)\right]^{\mathrm{GS}}, \quad \xi \in S^{1} \times S^{2 k-1},
$$

we have that for any $c \in \mathbb{R}$ and $\tau \in[c, c+1)$,

$$
\widehat{g}_{\tau}^{c} \text { and } \widehat{g} \text { induce the same element in }\left[S^{1} \times S^{2 k-1}, W_{2 k+1,3 k}\right] \text {. }
$$

Now, if $\left(\mathcal{N}_{1}, F_{1}\right)$ and $\left(\mathcal{N}_{2}, F_{2}\right)$ are TRC cobordant, then $F$ exists so that (4.2) holds on $\mathbf{T}$. Then, $(\tau, \theta, \eta) \mapsto\left(F_{*} \sigma_{\mathbf{T}}\right)(F(\tau, \theta, \eta)), 0 \leq \tau \leq 1-\delta$ is a homotopy between $\widehat{F_{1}}$ and $\widehat{F_{2}}$ in $W_{2 k+1,3 k}$.

To prove the converse, we rely on the relative $h$-principle for ample differential relations ([12, Theorem 18.4.1]). For this, we first gather some important observations about the relevant spaces. Wherever possible, we follow the notation employed in [12].

(1) Let $J^{1}\left(\mathbf{T}, \mathbb{C}^{3 k}\right)$ denote the space of 1 -jets of smooth maps from $\mathbf{T}$ into $\mathbb{C}^{3 k} \cong \mathbb{R}^{6 k}$. Since $\mathbf{T}$ is parallelizable, we may write $J^{1}\left(\mathbf{T}, \mathbb{R}^{6 k}\right) \cong \mathbf{T} \times \mathbb{C}^{3 k} \times M_{2 k+1,3 k}$, where $M_{2 k+1,3 k}$ is the space of complex $(2 k+1) \times(3 k)$ matrices. We use the field of frames $\sigma_{\mathbf{T}}$ to make this identification. This way, if $G: \mathbf{T} \rightarrow \mathbb{C}^{3 k}$ is a smooth map, then we have $D G(\cdot) \equiv\left(G_{*} \sigma_{\mathbf{T}}\right)(G(\cdot))$ (as matrices). Thus, $Z \mapsto\left(Z, G(Z),\left(G_{*} \sigma_{\mathbf{T}}\right)(G(Z))\right)$ is a holonomic section of $J^{1}\left(\mathrm{~T}, \mathbb{C}^{3 k}\right)$.

(2) We recall that $W_{2 k+1,3 k} \subset V_{2 k+1,3 k} \subset M_{2 k+1,3 k}$, and if $A, B \in V_{2 k+1,3 k}$, then any homotopy between $[A]^{\mathrm{GS}}$ and $[B]^{\mathrm{GS}}$ in $W_{2 k+1,3 k}$ lifts to a homotopy in $V_{2 k+1,3 k}$.

(3) We let $\mathcal{R}_{\mathrm{TR}}$ denote the differential relation in $J^{1}\left(\mathbf{T}, \mathbb{C}^{3 k}\right)$ corresponding to totally real immersions $f: \mathbf{T} \rightarrow \mathbb{C}^{3 k}$. Then $\mathcal{R}_{\mathrm{TR}}$ is an open ample differential relation (see for instance [12, Theorem 19.3.1]).

The relative $h$-principle states that if $\mathfrak{H}: \mathbf{T} \rightarrow \mathcal{R}_{\mathrm{TR}} \subset J^{1}\left(\mathbf{T}, \mathbb{C}^{3 k}\right)$ is a formal solution of $\mathcal{R}_{\mathrm{TR}}$ that is a genuine solution near $\partial \mathbf{T}$, then there is a homotopy of formal solutions $\mathfrak{H}_{\tau}: \mathbf{T} \rightarrow \mathcal{R}_{\mathrm{TR}}, \tau \in[0,1]$, joining $\mathfrak{H}_{0}=\mathfrak{H}_{\mathrm{H}}$ with a genuine solution $\mathfrak{S}_{1}$ of $\mathcal{R}_{\mathrm{TR}}$ such that for all $\tau, \mathfrak{H}_{\tau}=\mathfrak{H}$ near $\partial \mathbf{T}$.

To complete the proof of our claim, we note that if $\left(\mathcal{N}_{1}, F_{1}\right)$ and $\left(\mathcal{N}_{2}, F_{2}\right)$ are disjoint parametrized tubes enclosing curves in $\mathbb{C}^{3 k}$, then there exists a smooth map $G: \mathbf{T} \rightarrow \mathbb{C}^{3 k}$ such that

$$
G= \begin{cases}\left(F_{1} \circ L_{0}\right) & \text { on } \mathbf{T}_{\delta}, \\ \left(F_{2} \circ L_{1-\delta}\right) & \text { on } \mathbf{T}_{1-\delta},\end{cases}
$$

for $\delta \in\left(0, \frac{1}{2}\right)$ small enough. For this, we fix a $\delta \ll \frac{1}{2}$ and apply the isotopy version of the Weak Whitney Embedding Theorem to produce an isotopy between $\left(F_{1} \circ L_{0}\right)(\mathbf{T} \cap\{\tau=\delta\})$ and $\left(F_{2} \circ L_{1-\delta}\right)(\mathbf{T} \cap\{\tau=1-\delta\})$. This isotopy continuously connects $F_{1} \circ L_{0}$ on $\mathbf{T}_{\delta}$ and $F_{2} \circ L_{1-\delta}$ on $\mathbf{T}_{1-\delta}$ to give a continuous map $\widetilde{G}: \mathbf{T} \rightarrow \mathbb{C}^{3 k}$ that satisfies (4.3). Now, by the relative version of the Whitney Approximation Theorem, $\widetilde{G}$ can be approximated by a smooth $G$ satisfying (4.3).

Now, suppose $\widehat{F_{1}}$ and $\widehat{F_{2}}$ induce the same homotopy class in $\left[S^{1} \times S^{2 k-1}, W_{2 k+1,3 k}\right]$. In view of (b) and $(\dagger)$ above, there is a homotopy $H:[\delta, 1-\delta] \times S^{1} \times S^{2 k-1} \rightarrow V_{2 k+1,3 k}$

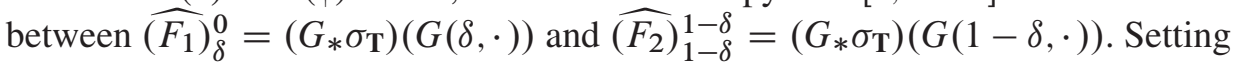

$$
\widetilde{H}: \mathbf{T} \rightarrow V_{2 k+1,3 k}
$$


as

$$
\widetilde{H}(\tau, \xi)= \begin{cases}\left(G_{*} \sigma_{\mathbf{T}}\right)(G(\tau, \xi) & \text { on } \mathbf{T}_{\delta}, \\ H(\tau, \xi) & \text { when } \tau \in[\delta, 1-\delta], \\ \left(G_{*} \sigma_{\mathbf{T}}\right)(G(\tau, \xi)) & \text { on } \mathbf{T}_{1-\delta}\end{cases}
$$

Then $\mathfrak{H}:(\tau, Z) \mapsto(Z, G(Z), \widetilde{H}(\tau, Z))$ is a formal solution of $\mathcal{R}_{\mathrm{TR}}$ that is a genuine solution near $\partial \mathbf{T}$. Thus, by the $h$-principle cited above, there is a genuine solution of $\mathcal{R}_{\mathrm{TR}}$ that coincides with $\mathfrak{S}$ near $\partial \mathbf{T}$, which gives a totally real immersion $\widetilde{F}: \mathbf{T} \rightarrow \mathbb{C}^{3 k}$ satisfying (4.2). Now, by another application of the (relative) Whitney Embedding Theorem, $\widetilde{F}$ can be approximated by a totally real embedding satisfying (4.2).

The above lemma shows that the TRC cobordism class of any parametrized tube $(\mathcal{N}, F)$ enclosing a curve in $\mathbb{C}^{3 k}$ contains all tubes of the form $(A(\mathcal{N}), F \circ A)$, where $A: \mathbb{C}^{3 k} \rightarrow \mathbb{C}^{3 k}$ is of the form $z \mapsto r z+b, r>0$ and $b \in \mathbb{C}^{3 k}$.

4.3. Polynomially convex models. So far, we have shown that there is an injective map

$$
\left\{\begin{array}{l}
\text { equivalence classes of parametrized tubes } \\
\text { enclosing curves under TRC cobordism }
\end{array}\right\} \hookrightarrow\left[S^{1} \times S^{2 k-1}, W_{2 k+1,3 k}\right] \text {. }
$$

In this subsection, we establish the surjectivity of this map. In fact, we construct polynomially convex representatives of the generators of $\left[S^{1} \times S^{2 k-1}, W_{2 k+1,3 k}\right]$, and then indicate how tubes of other indices can be obtained in a similar way. Our construction is a modification of the Beloshapka-Coffman normal form (2.1).

Consider the following set in $\mathbb{C} \times \mathbb{R}^{2 k-1}$ :

$$
\begin{aligned}
S=\left\{\left(z_{1}, x_{2}, \ldots, x_{2 k}\right) \in \mathbb{C} \times \mathbb{R}^{2 k-1}:\left|z_{1}\right|^{2}\right. & +\left(x_{3}\right)^{2}+\cdots+\left(x_{2 k-1}\right)^{2} \\
& \left.+\left(\sqrt{x_{2}^{2}+x_{2 k}^{2}}-1\right)^{2} \leq \frac{1}{4}\right\} .
\end{aligned}
$$

Note that $S$ is a neighborhood of the unit circle in the $x_{2} x_{2 k}$-plane. Now, consider the following two graphs over $S$ in $\mathbb{C}^{3 k}$ :

$$
\begin{aligned}
\mathcal{M}^{1}:=\left\{Z \in \mathbb{C}^{3 k}:\right. & \left(z_{1}, x_{2}, \ldots, x_{2 k}\right) \in S, y_{j}=0,2 \leq j \leq 2 k, \\
& z_{2 k+1}={\overline{z_{1}}}^{2}, \\
& z_{2 k+2}=\left|z_{1}\right|^{2}+\overline{z_{1}}\left(\sqrt{x_{2}^{2}+x_{2 k}^{2}}-1\right)+i \overline{z_{1}} x_{3}, \\
& \left.z_{\ell}=\overline{z_{1}}\left(x_{2(\ell-2 k-1)}+i x_{2(\ell-2 k-1)+1}\right), 2 k+3 \leq \ell \leq 3 k\right\}
\end{aligned}
$$

and

$$
\begin{aligned}
\mathcal{M}^{2}:=\left\{Z \in \mathbb{C}^{3 k}:\right. & \left(z_{1}, x_{2}, \ldots, x_{2 k}\right) \in S, y_{j}=0,2 \leq j \leq 2 k, \\
& z_{2 k+1}={\overline{z_{1}}}^{2}\left(x_{2}+i x_{2 k}\right), \\
& z_{2 k+2}=\left|z_{1}\right|^{2}+\overline{z_{1}}\left(\sqrt{x_{2}^{2}+x_{2 k}^{2}}-1\right)+i \overline{z_{1}} x_{3}, \\
& \left.z_{\ell}=\overline{z_{1}}\left(x_{2(\ell-2 k-1)}+i x_{2(\ell-2 k-1)+1}\right), 2 k+3 \leq \ell \leq 3 k\right\} .
\end{aligned}
$$


Since $\mathcal{M}^{1}$ and $\mathcal{M}^{2}$ are totally real except along the circle

$$
\gamma=\left\{\left(0, x_{2}, 0, \ldots, 0, x_{2 k}, 0, \ldots, 0\right) \in \mathbb{C}^{3 k}: x_{2}, x_{2 k} \in \mathbb{R}, x_{2}^{2}+x_{2 k}^{2}=1\right\},
$$

where they have CR dimension 1, they are tubes enclosing the CR-singular curve $\gamma$. We now prove the crucial fact the $\mathcal{M}_{1}$ and $\mathcal{M}_{2}$ are polynomially convex subsets of $\mathbb{C}^{3 k}$. As a side note, we also observe, that they are biholomorphically equivalent.

Lemma 4.8. There is a biholomorphism $\Theta$ defined in a neighborhood of $\mathcal{M}^{1}$ in $\mathbb{C}^{3 k}$ such that $\mathcal{M}^{2}=\Theta\left(\mathcal{M}^{1}\right)$. Moreover, $\gamma, \mathcal{M}^{1}$ and $\mathcal{M}^{2}$ are polynomially convex.

Proof. For the first part of the claim, consider the map

$$
\begin{aligned}
\Theta:\left(z_{1}, \ldots, z_{2 k}, z_{2 k+1}, z_{2 k+2}, \ldots, z_{3 k}\right) & \\
& \mapsto\left(z_{1}, \ldots, z_{2 k}, z_{2 k+1}\left(z_{2}+i z_{2 k}\right), z_{2 k+2}, \ldots, z_{3 k}\right),
\end{aligned}
$$

which is a biholomorphism from $\mathbb{C}^{3 k} \backslash\left\{z_{2}+i z_{2 k}=0\right\}$ onto itself. Clearly, $\Theta\left(\mathcal{M}^{1}\right)=\mathcal{M}^{2}$. Since $\mathcal{M}^{1}$ is a compact subset of $\mathbb{C}^{3 k} \backslash\left\{z_{2}+i z_{2 k}=0\right\}$, $\Theta$ is defined in a (sufficiently small) neighborhood of $\mathcal{M}^{1}$ in $\mathbb{C}^{3 k}$.

Now, for the second claim, we first note that the polynomial convexity of $\gamma$ follows from that fact that it is a compact subset of a totally real plane (the $x_{2} x_{2 k}$-plane). Next, we recall the following criterion (an iterated version of Theorem 1.2.16 from [30]). If $X \subset \mathbb{C}^{n}$ is a compact subset and if $F: X \rightarrow \mathbb{R}^{m}$ is a map whose components are in $\mathcal{P}(X)$, then $X$ is polynomially convex if and only if $F^{-1}(\boldsymbol{t})$ is polynomially convex for each $\boldsymbol{t} \in \mathbb{R}^{m}$. Now, choosing the restriction to $M^{j}$ of the map $F: \mathbb{C}^{3 k} \rightarrow \mathbb{C}^{2 k-1}$ given by $Z \mapsto\left(z_{2}, \ldots, z_{2 k}\right)$, and noting that the subalgebra generated by $z$ and $c \bar{z}^{2}, c \neq 0$, in $\zeta\left(\frac{1}{2} \overline{\mathbb{D}}\right)$ coincides with $\zeta\left(\frac{1}{2} \overline{\mathbb{D}}\right)$ (see [26]), we have that every fibre of $F$ in either $M^{1}$ or $M^{2}$ is polynomially convex. Hence, the claim. by

We now fix parametrizations of $\mathcal{M}^{1}$ and $\mathcal{M}^{2}$. Let $\iota: S^{1} \times D^{2 k} \rightarrow S$ be the map given

$$
\begin{gathered}
\iota:(\theta, z, t, \zeta, s) \mapsto\left(\frac{1}{2} x,-\frac{1}{2} y,\left(1+\frac{1}{2} t\right) \cos \theta, \frac{1}{2} s, \frac{1}{2} u_{1}, \frac{1}{2} v_{1}, \ldots, \frac{1}{2} u_{k-2},\right. \\
\left.\frac{1}{2} v_{k-2},\left(1+\frac{1}{2} t\right) \sin \theta\right) .
\end{gathered}
$$

As each $M^{j}$ is a graph over $S$, the parametrization of $S$ can be pushed forward via the graphing map to obtain a parametrizing map of $M^{j}$, which we denote by $H^{j}, j=1,2$.

Theorem 4.9. Let $k \geq 2$. Then, $\operatorname{ind}_{H^{1}}\left(\mathcal{M}^{1}\right)=\operatorname{ind}_{H^{2}}\left(\mathcal{M}^{2}\right)=1$. Moreover:

(i) when $k$ is even, $\left[\widehat{H}_{1}\right]=\left[\widehat{H}_{2}\right]$ in $\left[S^{1} \times S^{2 k-1}, W_{2 k+1,3 k}\right]$, but

(ii) when $k$ is odd, $\left[\widehat{H}_{1}\right] \neq\left[\widehat{H}_{2}\right]$ in $\left[S^{1} \times S^{2 k-1}, W_{2 k+1,3 k}\right]$.

Proof. As before, since $W_{2 k+1,3 k}$ is a deformation retract of $V_{2 k+1,3 k}$, we ignore the effect of $[\cdot]^{\mathrm{GS}}$ in the definitions of $\widehat{H}^{j}$ and $\widehat{H}_{\text {slice }}^{j}, j=1,2$. Now, due to Lemmas 3.3 and 3.4, it suffices to show that $\left[\widehat{H}^{1}\right]=\left[f_{2}\right]$ and $\left[\widehat{H}^{2}\right]=\left[f_{2}\right]$ in $\left[S^{1} \times S^{2 k-1}, W_{2 k+1,3 k}\right]$, where $f_{1}$ and $f_{2}$ are the maps defined in Lemma 3.4. We describe a homotopy between $\widehat{H}^{1}$ and $f_{1}$ in some detail, and note that an almost identical procedure gives a homotopy between $\widehat{H}^{2}$ and $f_{2}$. 
Recall that we use the coordinates

$$
\xi=\left(\theta, z=x+i y, t, \zeta_{1}=u_{1}+i v_{1}, \ldots, \zeta_{k-2}=u_{k-2}+i v_{k-2}, s\right)
$$

on $S^{1} \times S^{2 k-1}$. Up to a cyclical permutation of the columns (which preserves the homotopy classes of $V_{2 k+1,3 k}$ ), we may write $\widehat{H}^{1}(\xi)=D H^{1}(\xi)$ explicitly as follows:

$$
\begin{aligned}
& \left(E_{3 k \times 2} \mid F_{3 k \times 2 k-1}\right)=\left(\begin{array}{c|c}
A_{2 k \times 2} & \frac{B_{2 k \times 2 k-1}}{C_{k \times 2}} \\
D_{k \times 2 k-1}
\end{array}\right)
\end{aligned}
$$

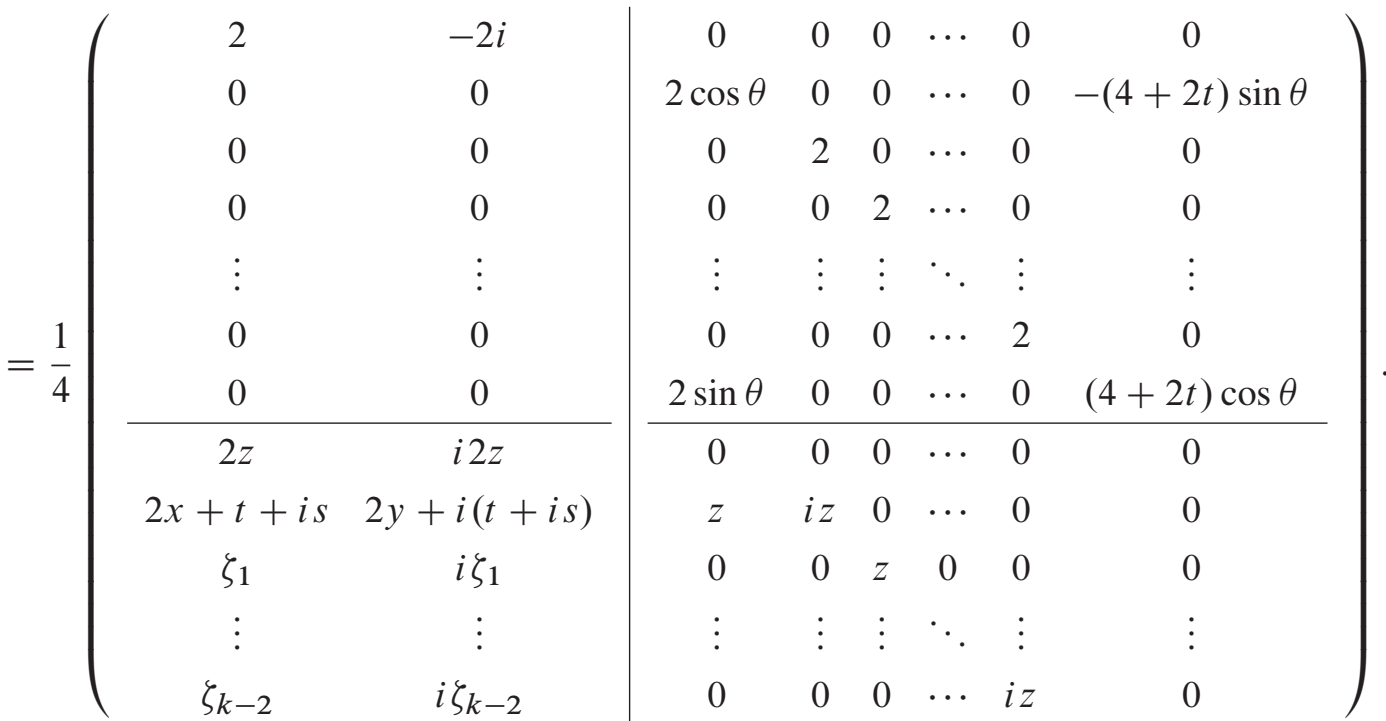

For any matrix $M_{p \times q}$ above, $\operatorname{span}_{\mathbb{C}}[M]$ will denote the complex span of its $q$ columns in $\mathbb{C}^{p}$. Note that if $F_{t}$ is a homotopy of $F$ through $(3 k) \times(2 k-1)$ matrices of full rank such that $\operatorname{span}_{\mathbb{C}}\left[F_{t}\right] \oplus \operatorname{span}_{\mathbb{C}}[E]=\mathbb{C}^{3 k}$, then $\left(E \mid F_{t}\right)$ is a homotopy of $(E \mid F)$ in $V_{2 k+1,3 k}$. With this principle in mind, we will perform the homotopy in multiple steps. First, we get rid of the factor $\frac{1}{4}$ via an elementary homotopy.

(a) We note that the matrix $D$ has no impact on the rank of $(E \mid F)$. Thus, the following homotopy takes place within $V_{2 k+1,3 k}$ :

$$
(\tau, \xi) \mapsto\left(\frac{A}{C} \mid \frac{B}{(1-\tau) D}\right), \quad \tau \in[0,1] .
$$

Thus, we assume here onwards that $D=\mathbf{0}$.

(b) With $D=\mathbf{0}, \operatorname{span}[F]=\operatorname{span}\left\{z_{2}, \ldots, z_{2 k}\right\} \cong \mathbb{C}^{2 k-1}$ is orthogonal to $\operatorname{span}[E]$. Thus, if $B^{\prime} \in \mathrm{GL}_{\mathbb{C}}(2 k-1)$ denotes the matrix obtained by deleting the first row of $B$, any homotopy $B_{t}^{\prime}$ of $B^{\prime}$ in $\mathrm{GL}_{\mathbb{C}}(2 k-1)$ induces a homotopy of $(E \mid F)$ in $V_{2 k+1,3 k}$. Note that $B^{\prime}$ only depends on $(t, \theta) \in[-1,1] \times S^{1}$, and $\frac{2^{-2 k}}{4+2 t} B^{\prime}(\xi)$ is an element in $\operatorname{SU}(2 k-1)$. Thus, we have that $\xi \mapsto \frac{2^{-2 k}}{4+2 t} B^{\prime}(\xi)$ induces an element in $\pi_{1}(\mathrm{SU}(2 k-1)) \cong 0$. So, there is a homotopy between $\xi \mapsto B^{\prime}(\xi)$ and $\xi \mapsto I$ in $\operatorname{GL}_{\mathbb{C}}(2 k-1)$. We may now assume that

$$
B=\left(\frac{\mathbf{0}_{1,2 k-1}}{\mathbf{I}_{2 k-1,2 k-1}}\right) \text {. }
$$


(c) Since $\operatorname{span}[E]=\operatorname{span}\left\{z_{1}, z_{2 k+1}, \ldots, z_{3 k}\right\} \cong \mathbb{C}^{k}$ is orthogonal to $\operatorname{span}[F]$, it suffices to produce an appropriate homotopy in $V_{2, k+1}$ of

$$
E^{\prime}=\left(\begin{array}{cc}
2 & -2 i \\
2 z & i 2 z \\
2 x+t+i s & 2 y+i(t+i s) \\
\zeta_{1} & i \zeta_{1} \\
\vdots & \vdots \\
\zeta_{k-2} & i \zeta_{k-2}
\end{array}\right)
$$

which, after an elementary homotopy in $V_{2, k+1}$, becomes

$$
\left(\begin{array}{cc}
0 & -i \\
z & i z \\
t+i s & i(t+i s) \\
\zeta_{1} & i \zeta_{1} \\
\vdots & \vdots \\
\zeta_{k-2} & i \zeta_{k-2}
\end{array}\right)=:\left(E_{1} \mid E_{2}\right)
$$

Now, the homotopy

$$
E_{\tau}^{\prime}=\left(E_{1} \mid e^{i \tau \frac{\pi}{2}} E_{2}+i \tau E_{1}\right), \quad \tau \in[0,1],
$$

gives that $\widehat{H}^{1}$ is homotopic to

$$
\xi \mapsto \widetilde{i}_{2 k}(z, t, \zeta, s)=\left(\begin{array}{c|c}
\mathbf{0}_{2 k, 1} & \mathbf{I}_{2 k, 2 k} \\
z & \\
t+i s & \mathbf{0}_{k, 2 k} \\
\zeta^{T} &
\end{array}\right) .
$$

(d) Recall that $f_{1}(\xi)=i_{2 k}(z, y, \zeta, s)$, where $i_{2 k} \equiv E \cdot \widetilde{i}_{2 k}$ and $E$ is an elementary matrix in $\mathrm{GL}_{\mathbb{C}}(3 k)$ whose action on $V_{2 k+1,3 k}$ is to swap the lower block of $k$ rows with the upper block of $2 k$ rows. As $\mathrm{GL}_{\mathbb{C}}(3 k)$ is connected, we may homotope $\widetilde{i}_{2 k}=E^{-1} \cdot i_{2 k}$ to $i_{2 k}=\mathbf{I} \cdot i_{2 k}$. Hence, our claim.

As noted earlier, a similar homotopy can be produced between $\widehat{H}^{2}$ and $f_{2}$.

Corollary 4.10. Suppose $\mathcal{N}$ is a tube enclosing a CR-singular curve $\gamma$ in $\mathbb{C}^{3 k}$ such that for some $p \in \gamma, \mathcal{N}$ at $p$ is locally biholomorphic to the Beloshapka-Coffman normal form $\mathscr{B}^{2 k+1,3 k}$ at 0 (see (2.1)). Then there exists an admissible parametrization $F$ of $\mathcal{N}$ such that $\operatorname{ind}_{F} \mathcal{N}=1$.

Proof. By the local biholomorphic invariance of the index (see Lemma 4.3), it suffices to show that the index of $\mathscr{B}^{2 k+1,3 k}$ at 0 is \pm 1 in the following sense. For $\varepsilon<2 \pi$, if $H:\left\{e^{i \theta}:|\theta|<\varepsilon\right\} \times D^{2 k} \rightarrow \mathbb{C}^{3 k}$ is a parametrization of some neighborhood $U \subset \mathscr{B}^{2 k+1,3 k}$ of 0 , which maps $(1,0)$ to 0 and the curve $\left\{e^{i \theta}:|\theta|<\varepsilon\right\} \times\{0\}$ onto $U \cap\left(x_{2 k}\right.$-axis $)$, then $\operatorname{deg}\left(\widehat{H}_{1 \text {-slice }}\right)= \pm 1$. Since the induced degree of any two such parametrizations will only differ by a sign (again, see Lemma 4.3 ), it suffices to produce some $H$ for which $\operatorname{deg}\left(\widehat{H}_{1 \text {-slice }}\right)=1$. 
This is essentially done in Theorem 4.9, since, up to translation,

$$
\mathcal{M}_{1} \cap\left\{x_{2 k}=0\right\}=\mathscr{B}^{2 k+1,3 k} \cap\left\{x_{2 k}=0,\left\|\left(z_{1}, x_{2}, \ldots, x_{2 k-1}\right)\right\|<\frac{1}{2}\right\} .
$$

To be more precise, we note that the map $h=\left(h_{1}, \ldots, h_{3 k}\right)$ given by

$$
h_{j}\left(z_{1}, \ldots, z_{3 k}\right)= \begin{cases}z_{j} & \text { if } j \neq 2,2 k, \\ \sqrt{z_{2}^{2}+z_{2 k}^{2}}-1 & \text { if } j=2, \\ \arctan \left(\frac{x_{2 k}}{x_{2}}\right) & \text { if } j=2 k,\end{cases}
$$

is a local biholomorphism near $p=(0,1,0, \ldots, 0) \in \mathcal{M}_{1}$ which maps a neighborhood of $p$ in $\mathcal{M}_{1}$ to a neighborhood of 0 in $\mathcal{B}^{2 k+1,2 k}$. The local biholomorphic invariance of the index implies that $H=h \circ H^{1}$, where $H^{1}$ is as in Theorem 4.9, is the desired parametrization of $B^{2 k+1,3 k}$ near 0 .

We now indicate how $\mathcal{M}_{1}$ and $\mathcal{M}_{2}$ can be modified to produce polynomially convex models of all indices. For any nonnegative index $n$, one can set $z_{2 k+1}$ as $\bar{z}_{1}^{n+1}$ and $\bar{z}_{1}^{n+1}\left(x_{2}+i x_{2 k}\right)$ in the definitions of $\mathcal{M}^{1}$ and $\mathcal{M}^{2}$, respectively, and modify $H^{1}$ and $H^{2}$ in the obvious way. As in Theorem 4.9, these two models will represent the same and only TRC cobordism class of index $n$ when $k$ is even, and the only two distinct TRC cobordism classes of index $n$ when $k$ is odd. For tubes of negative indices, we note that if $(\mathcal{N}, F)$ is a parametrized tube of index $n, n \in \mathbb{N}$, then $\left(\mathcal{N}, F^{*}\right)$ is a parametrized tube of index $-n$, where $F^{*}:(\theta, \eta) \mapsto\left(\theta, \eta^{*}\right)$, $(\theta, \eta) \in S^{1} \times D^{2 k}$ (see Remark 4.4).

4.4. A fully-TR tube containing an Alexander set. At the end of Section 4.3, we construct polynomially convex parametrized tubes of index zero. These are in fact fully-TR tubes. In the proof of Theorem 1.2, we will use a fully-TR tube with an extremely different complex-analytic behavior from the ones mentioned above. This construction is based on the following result due to Alexander ([3]) which has been heavily used in the context of hulls without analytic structure (see [23] and [5]). The standard torus $\mathbb{T}^{2}=\left\{\left(e^{i \theta}, e^{i \psi}\right): \theta, \psi \in \mathbb{R}\right\}$ in $\mathbb{C}^{2}$ contains a compact subset $E$ such that $\widehat{E} \backslash E$ is nonempty but contains no analytic disk. Such a set can be chosen in any neighborhood of the diagonal of $\mathbb{T}^{2}$.

Now, for some $\varepsilon \ll 1$, let $F=\left(f_{1}, \ldots, f_{3 k}\right): S^{1} \times \varepsilon D^{2 k} \rightarrow \mathbb{C}^{3 k}$ be the smooth map given by

$$
\begin{gathered}
\left(f_{1}, \ldots, f_{2 k+1}\right):\left(\theta, x, y, t, u_{1}, v_{1}, \ldots, u_{2 k}, v_{2 k}, s\right) \\
\mapsto\left(e^{i \theta}, e^{i(\theta+s)}, x, y, t, u_{1}, v_{1}, \ldots, u_{2 k}, v_{2 k}\right), \\
f_{j} \equiv 0, \quad 2 k+2 \leq j \leq 3 k .
\end{gathered}
$$

Lemma 4.11. Let $\mathcal{A}:=F\left(S^{1} \times \varepsilon D^{2 k}\right)$ and $G:(\theta, \eta) \mapsto F(\theta, \varepsilon \eta),(\theta, \eta) \in S^{1} \times D^{2 k}$. Then $\mathcal{A}$ is a fully-TR tube in $\mathbb{C}^{3 k}$ and $[\widehat{G}]$ is trivial in $\left[S^{1} \times S^{2 k-1}, W_{2 k+1,3 k}\right]$. Moreover, A contains a compact set $E$ such that such that $\widehat{E} \backslash E$ is nonempty but contains no analytic disk.

Proof. The first part of the claim follows from computing $D G$ and observing that it depends only on $(\theta, s) \in S^{1} \times[-1,1]$. Since $\pi_{1}\left(W_{2 k+1,3 k}\right)$ is trivial, so is $[\widehat{G}]$. 
For the second part of the claim, note that $\mathcal{A}$ contains a neighborhood $V$ of the diagonal of the standard torus in the $z_{1}-z_{2}$ plane. In fact, $V=F\left(\left\{x=y=t=u_{j}=v_{j}=0\right\}\right)$. Note that if $E \subset V$ is the set granted by Alexander's theorem above, then $E \times\{0\}^{3 k-2}$ is a compact set in $\mathcal{A}$ with same hulls as $E$. Thus, in an abuse of notation, denoting $E \times\{0\}^{3 k-2}$ by $E$ completes the proof.

\section{Proofs of the main results}

We first isolate a lemma that is used in both the proofs in this section. The proof uses standard arguments; see [18, Lemma 2.1] for a version of this lemma. Here, for $p \in \mathbb{C}^{n}$ and $r>0, B_{p}(r)=\left\{z \in \mathbb{C}^{n}:\|z-p\|<r\right\}$ and $\bar{B}_{p}(r)$ denotes its closure in $\mathbb{C}^{n}$

Lemma 5.1. Suppose that $X \subset \mathbb{C}^{n}$ is a polynomially convex compact set and suppose $p_{1}, \ldots, p_{\ell} \in \mathbb{C}^{n} \backslash X$. Then there exist $r_{1}, \ldots, r_{\ell}>0$, so that $X \cup \cup_{j=1}^{\ell} Q_{j}$ is polynomially convex, for any choice of polynomially convex compact sets $Q_{j} \subseteq \bar{B}_{p_{j}}\left(r_{j}\right), j=1, \ldots, \ell$.

5.1. Proof of Theorem 1.1. Let $M$ be as given. In view of Lemma 2.2 and Corollary 4.10, we may assume that $M$ is embedded in $\mathbb{C}^{3 k}$, its set of CR-singularities is a union of disjoint simple closed curves, $C_{1}, \ldots, C_{\ell}$, and each $C_{j}$ is enclosed in a parametrized tube $\left(\mathcal{N}_{j}, F_{j}\right)$, where $\mathcal{N}_{j} \subset M$ and ind $F_{j}\left(\mathcal{N}_{j}\right)=1$. Using the results of Section 4 , we now proceed to "replace" the $\mathcal{N}_{j}$ 's with polynomially convex tubes enclosing CR-singular curves.

Lemma 5.2. There exist $\ell$ disjoint tubes, $\mathcal{K}_{1}, \ldots, \mathcal{K}_{\ell}$, enclosing $C R$-singular curves $\gamma_{1}, \ldots, \gamma_{\ell}$ such that

(i) each $\mathcal{K}_{j}$ is disjoint from $M$,

(ii) for some parametrization $G_{j},\left(\mathcal{K}_{j}, G_{j}\right)$ is TRC cobordant to $\left(\mathcal{N}_{j}, F_{j}\right), j=1, \ldots, \ell$,

(iii) $\Gamma=\gamma_{1} \cup \cdots \cup \gamma_{\ell}$ is polynomially convex in $\mathbb{C}^{3 k}$, and

(iv) $\mathcal{K}=\mathcal{K}_{1} \cup \cdots \cup \mathcal{K}_{\ell}$ is polynomially convex in $\mathbb{C}^{3 k}$.

Proof. First, since ind $F_{j}\left(\mathcal{N}_{j}\right)=1,\left(\mathcal{N}_{j}, F_{j}\right)$ is TRC cobordant to either $\left(\mathcal{M}^{1}, H^{1}\right)$ or $\left(\mathcal{M}^{2}, H^{2}\right)$ (which are TRC cobordant to each other when $k$ is even). This follows from the characterization of TRC cobordism classes obtained in Lemma 4.7. Without loss of generality, we assume that there is some $\ell_{0} \in\{1, \ldots, \ell\}$ such that $\left(\mathcal{N}_{1}, F_{1}\right), \ldots,\left(\mathcal{N}_{\ell_{0}}, F_{\ell_{0}}\right)$ are TRC cobordant to $\left(\mathcal{M}^{1}, H^{1}\right)$, and $\left(\mathcal{N}_{\ell_{0}+1}, F_{\ell_{0}+1}\right), \ldots,\left(\mathcal{N}_{\ell}, F_{\ell}\right)$ are TRC cobordant to $\left(\mathcal{M}^{2}, H^{2}\right)$.

Next, let $p_{1}, \ldots, p_{\ell} \in \mathbb{C}^{3 k} \backslash M$ be distinct points. Let $r_{1}, \ldots, r_{\ell}>0$ be as granted by Lemma 5.1 (for $X=\emptyset$ ). By shrinking the radii $r_{j}$ further, we may assume that each $\bar{B}_{p_{j}}\left(r_{j}\right)$ is disjoint from $M$. Now observe that the CR structure, polynomial convexity (both of the tubes and the enclosed CR-singular curves) and the TRC cobordism class of $\left(\mathcal{M}^{1}, H^{1}\right)$ and $\left(\mathcal{M}^{2}, H^{2}\right)$ remain unchanged under transformations of the form $A: z \mapsto r z-p$, where $r>0$ and $p \in \mathbb{C}^{3 k}$. For such a map $A$, we call $\left(A\left(\mathcal{M}^{j}\right), H^{j} \circ A\right)$ a copy of $\left(\mathcal{M}^{j}, H^{j}\right)$, and it is a parametrized tube in $B_{p}(r)$ enclosing the CR-singular curve $A(\gamma)$.

Let $\left(K_{1}, G_{1}\right), \ldots,\left(K_{\ell_{0}}, G_{\ell_{0}}\right)$ be copies of $\left(\mathcal{M}_{1}, H^{1}\right)$ in $B_{p_{1}}\left(r_{1}\right), \ldots, B_{p_{\ell_{0}}}\left(r_{\ell_{0}}\right)$, respectively, and $\left(K_{\ell_{0}+1}, G_{\ell_{0}+1}\right), \ldots,\left(K_{\ell}, G_{\ell}\right)$ copies of $\left(\mathcal{M}_{2}, H^{2}\right)$ in $B_{p_{\ell_{0}+1}}\left(r_{\ell_{0}+1}\right), \ldots, B_{p_{\ell}}\left(r_{\ell}\right)$, respectively. Denote by $\gamma_{j}$ the CR-singular curve enclosed by $K_{j}, j=1, \ldots, \ell$. 
Finally, note that (i) and (ii) hold by construction. Since $K_{1}, \ldots, K_{\ell}$ and $\gamma_{1}, \ldots, \gamma_{\ell}$ are polynomially convex subsets of $\mathbb{C}^{3 k}$ contained in the balls $B_{p_{1}}\left(r_{1}\right), \ldots, B_{p_{\ell}}\left(r_{\ell}\right)$, respectively, Lemma 5.1 gives (iii) and (iv). This concludes the proof of Lemma 5.2.

Fix a $j \in\{1, \ldots, \ell\}$. By the neighborhood replacement result, i.e., Proposition 4.6, there is a smooth embedding

$$
\Psi_{\mathcal{N}_{j}, K_{j}}: S^{1} \times 2 D^{2 k} \rightarrow \mathbb{C}^{3 k}
$$

such that if we set

$$
\mathcal{T}_{j}:=\Psi_{\mathcal{N}_{j}, K_{j}}\left(S^{1} \times 2 D^{2 k}\right)
$$

then

(a) $\partial \mathcal{T}_{j}=\partial \mathcal{N}_{j}$,

(b) a collar of $\partial \mathcal{T}_{j}$ in $\mathcal{T}_{j}$, say coll $\left(\partial \mathcal{T}_{j}\right)$, coincides with a collar of $\partial \mathcal{N}_{j}$ in $\mathcal{N}_{j}$,

(c) $K_{j} \subset \mathcal{T}_{j}$, and $\mathcal{T}_{j}$ is totally real everywhere except along $\gamma_{j} \subset K_{j}$.

Let $\mathcal{N}_{j}^{\prime} \subset \mathcal{N}_{j}$ be a slightly thinner tubular neighborhood of $C_{j}$ chosen so that $\partial \mathcal{N}_{j}^{\prime} \subset \operatorname{coll}\left(\partial \mathcal{T}_{j}\right)$. Now, letting $M^{*}=M \backslash\left(\mathcal{N}_{1}^{\prime} \cup \cdots \cup \mathcal{N}_{\ell}^{\prime}\right)$, we set

$$
M_{1}=M^{*} \cup\left(\bigcup_{j=1}^{\ell} \tau_{j}\right) \text {. }
$$

We note the inclusion map $\iota: M_{1} \rightarrow \mathbb{C}^{3 k}$ is an immersion of $M$ whose image is totally real except along $\Gamma=\gamma_{1} \cup \cdots \cup \gamma_{\ell}$, which admits a polynomially convex neighborhood $K$ in $M_{1}$. Moreover, $\iota$ is a smooth embedding when restricted to a sufficiently small open neighborhood of $K$ in $M_{1}$. Thus, by the relative version of the weak Whitney embedding theorem, we may perturb $\iota$, keeping it fixed over $K$, to obtain a smooth embedding $j: M \rightarrow \mathbb{C}^{3 k}$ such that $K \subset j(M)$, and $j(M) \backslash \Gamma$ is totally real.

Note that $j(M) \backslash K^{\circ}$ is a compact totally real smooth submanifold of $\mathbb{C}^{3 k}$ with boundary, and $K$ is polynomially convex. Thus, we can apply the following result due to Arosio and Wold (see [5, Theorem 1.4]).

Theorem. Let $N$ be a compact smooth manifold (possibly with boundary) of dimension $d<n$ and let $f: N \rightarrow \mathbb{C}^{n}$ be a totally real $e^{\infty}$-embedding. Let $X \subset \mathbb{C}^{n}$ be a compact polynomially convex set. Then, for all $s \geq 1$ and for all $\varepsilon>0$, there exists a totally real $e^{\infty}$-embedding $f_{\varepsilon}: N \rightarrow \mathbb{C}^{n}$ such that

(a) $\left\|f-f_{\varepsilon}\right\| \varphi^{s}(N)<\varepsilon$,

(b) $f_{\varepsilon}=f$ on $f^{-1}(X)$, and

(c) $\overline{X \cup f_{\varepsilon}(N)}=X \cup f_{\varepsilon}(N)$.

We set $N=M \backslash j^{-1}\left(K^{\circ}\right), X=K$ and $f=\left.j\right|_{N}$, and let $\varepsilon>0$ be arbitrary but fixed. Then $M^{\prime}=f_{\varepsilon}\left(N \backslash j^{-1}(K)\right) \cup K$ is an embedded submanifold of $\mathbb{C}^{3 k}$ that is diffeomorphic to $M$, polynomially convex, and totally real away from $\Gamma \subset M^{\prime}$, which is a union of finitely many disjoint simple closed curves. This gives items (1) and (2) of the theorem.

It remains to show that $\mathcal{C}\left(M^{\prime}\right)=\mathscr{P}\left(M^{\prime}\right)$. For this, we use the following result due to O'Farrel-Preskenis-Walsch; see [28] or [30, Section 6.3]. 
Theorem. Let $X$ be a compact holomorphically convex set in $\mathbb{C}^{n}$, and let $X_{0}$ be a closed subset of $X$ for which $X \backslash X_{0}$ is a totally real subset of the manifold $\mathbb{C}^{n} \backslash X_{0}$. A function $f \in \mathcal{C}(X)$ can be approximated uniformly on $X$ by functions holomorphic on a neighborhood of $X$ if and only if $\left.f\right|_{X_{0}}$ can be approximated uniformly on $X_{0}$ by functions holomorphic on $X$.

First, we apply the above result (or an earlier version by Harvey-Wells; see [19]) to $X=\Gamma$ and $X_{0}=\emptyset$ to obtain that any $f \in \mathscr{C}(\Gamma)$ can be approximated uniformly on $\Gamma$ by functions holomorphic on a neighborhood of $\Gamma$. Since $\Gamma$ is polynomially convex, the Oka-Weil theorem allows us to conclude that $\mathscr{C}(\Gamma)=\mathcal{P}(\Gamma)$. Thus, $X:=M^{\prime}$ and $X_{0}=\Gamma$ satisfy the hypothesis of the O'Farrel-Preskenis-Walsch result, and any $f \in \mathscr{C}^{\prime}\left(M^{\prime}\right)$ can be approximated uniformly on $M^{\prime}$ by functions holomorphic on a neighborhood of $M^{\prime}$. Once again, applying the Oka-Weil theorem to the polynomially convex set $M^{\prime}$, we obtain that $C^{\prime}\left(M^{\prime}\right)=\mathcal{P}\left(M^{\prime}\right)$.

5.2. Proof of Theorem 1.2. We let $\iota: M \rightarrow \mathbb{C}^{3 k}$ be the embedding granted by Theorem 1.1. Recall from the proof of the theorem that the CR-singular set $\Gamma$ of $\iota(M)$ is contained in a polynomially convex compact set $K \subset \iota(M)$. Now, by Lemma 5.1, we can find a small closed ball $Y \subset \mathbb{C}^{3 k}$ that is disjoint from $\iota(M)$ and is such that $K \cup Q$ is polynomially convex for any polynomially convex subset $Q \subset Y$. We now find a copy of $\mathcal{A}-$ the tube constructed in Section 4.4 - in the ball $Y$, by using an appropriate map of the form $z \mapsto r z-p, r>0$, $p \in \mathbb{C}^{3 k}$. We abuse notation and call this copy and the Alexander set contained in it $\mathcal{A}$ and $E$, respectively. Since $\widehat{E} \subset Y, K \cup \widehat{E}$ is polynomially convex.

Now, let $\mathcal{N}$ be a fully-TR tube in some small totally real ball $B \subset \iota(M)$. Then, by taking any parametrization of $B$ by $D^{2 k+1}$ and restricting it to a copy of $S^{1} \times D^{2 k}$ in $D^{2 k+1}$, we obtain an admissible parametrization $\mathcal{N}$ whose frame map induces the trivial element in $\left[S^{1} \times S^{2 k-1}, W_{2 k+1,3 k}\right]$. Using the neighborhood replacement result and Lemma 4.11, we replace $\mathcal{N}$ by $\mathcal{A}$. Combined with the relative version of the weak Whitney Embedding Theorem, this gives an embedding $j: M \rightarrow \mathbb{C}^{3 k}$ such that

- $j$ coincides with $\iota$ on $\iota^{-1}(K)$,

- $j(M) \backslash \Gamma$ is totally real, and

- there is a totally real ball $B^{\prime} \subset j(M)$ that contains $\mathcal{A}$.

We now apply the Aroiso-Wold perturbation result stated in Section 5.1 to $N=M \backslash j^{-1}(K)$, $X=\widehat{E} \cup K$ and $\left.j\right|_{N}$. We obtain a smooth embedding of $M$ into $\mathbb{C}^{3 k-1}$ whose image $\Sigma$ contains $E \cup K$, is totally real away from $\Gamma$, and

$$
\widehat{\Sigma \cup \widehat{E}}=\Sigma \cup \widehat{E} \text {. }
$$

Thus, $\widehat{\Sigma}=\widehat{\Sigma \cup E}=\Sigma \cup \widehat{E}$. Now, if $\widehat{E}$ was contained in $\Sigma$, then $\Sigma$ would be a polynomially convex manifold that is totally real except along the polynomially convex set $\Gamma$. This is precisely the situation handled in the proof of Theorem 1.1 using the O'Farrel-Preskenis-Walsch result cited above. Using the same technique, we see that any subset $T$ of such a manifold has the property that $\mathcal{C}(T)=\mathcal{P}(T)$, and thus is polynomially convex. This contradicts the fact that $E \subset \Sigma$ is not polynomially convex. Thus, $\widehat{\Sigma} \backslash \Sigma$ is nonempty but contains no analytic disk.

To show that $\widehat{\Sigma}=h_{r}(\Sigma)$, we use an argument due to Izzo, who showed in [22, Section 3] that $E$ satisfies the generalized argument principle, i.e., if $p$ is a polynomial that has a continuous logarithm on $E$, then $0 \notin p(E)$. We now apply the following result due to Stolzenberg ([29]) to $X=E$ and $Y=B^{\prime}$. 
Theorem. If $X \subseteq Y \subset \mathbb{C}^{n}$ are compact sets such that $X$ satisfies the generalized argument principle and the first $\check{C}$ ech cohomology group $\check{\mathrm{H}}^{1}(Y, \mathbb{Z})$ vanishes, then $\widehat{X} \subset h_{r}(Y)$.

Thus, $\widehat{\Sigma}=\Sigma \cup \widehat{E} \subseteq h_{r}(\Sigma)$, and the two hulls coincide.

\section{References}

[1] J. F. Adams, Stable homotopy and generalised homology, Chicago Lectures in Math. University of Chicago Press, Chicago 1974.

[2] H. Alexander, Linking and holomorphic hulls, J. Differential Geom. 38 (1993), no. 1, 151-160.

[3] H. Alexander, Hulls of subsets of the torus in $\mathbb{C}^{2}$, Ann. Inst. Fourier (Grenoble) 48 (1998), no. 3, 785-795.

[4] M. Arkowitz, Introduction to homotopy theory, Universitext, Springer, New York 2011.

[5] L. Arosio and E. F. Wold, Totally real embeddings with prescribed polynomial hulls, Indiana Univ. Math. J. 68 (2019), no. 2, 629-640.

[6] V.K. Beloshapka, The normal form of germs of four-dimensional real submanifolds in $\mathbb{C}^{5}$ at generic $\mathbb{R} \mathbb{C}$-singular points, Math. Notes 61 (1997), no. 5, 777-779.

[7] E. Bishop, Differentiable manifolds in complex Euclidean space, Duke Math. J. 32 (1965), 1-21.

[8] K. Cieliebak and Y. Eliashberg, The topology of rationally and polynomially convex domains, Invent. Math. 199 (2015), no. 1, 215-238.

[9] A. Coffman, Enumeration and normal forms of singularities in Cauchy-Riemann structures, ProQuest LLC, Ann Arbor 1997; Ph.D. thesis, The University of Chicago, Chicago 1997.

[10] A. Coffman, Unfolding CR singularities, Mem. Amer. Math. Soc. 205 (2010), no. 962.

[11] A. V. Domrin, A description of characteristic classes of real submanifolds in complex manifolds in terms of RC-singularities, Izv. Math. 59 (1995), no. 5, 899-918.

[12] Y. Eliashberg and N. Mishachev, Introduction to the h-principle, Grad. Stud. Math. 48, American Mathematical Society, Providence 2002.

[13] F. Forstnerič, Approximation by automorphisms on smooth submanifolds of $\mathbf{C}^{n}$, Math. Ann. 300 (1994), no. 4, 719-738.

[14] F. Forstnerič, Complements of Runge domains and holomorphic hulls, Michigan Math. J. 41 (1994), no. 2, 297-308.

[15] F. Forstnerič and J.-P. Rosay, Approximation of biholomorphic mappings by automorphisms of $\mathbf{C}^{n}$, Invent. Math. 112 (1993), no. 2, 323-349.

[16] M. E. Gilmore, Complex Stiefel manifolds, some homotopy groups and vector fields, Bull. Amer. Math. Soc. 73 (1967), 630-633.

[17] M. E. Gilmore, Homotopy groups of complex Stiefel manifolds, ProQuest LLC, Ann Arbor 1967; Ph.D. thesis, University of California, Berkeley 1967.

[18] P. Gupta and R. Shafikov, Polynomially convex embedddings of even-dimensional compact manifolds, Ann. Sc. Norm. Super. Pisa Cl. Sci. (5) 21 (2020), 1649-1666.

[19] F. R. Harvey and R. O. Wells, Holomorphic approximation on totally real submanifolds of a complex manifold, Bull. Amer. Math. Soc. 77 (1971), no. 5, 824-828.

[20] A. Hatcher, Algebraic topology, Cambridge University Press, Cambridge 2002.

[21] P. T. Ho, H. Jacobowitz and P. Landweber, Optimality for totally real immersions and independent mappings of manifolds into $\mathbb{C}^{N}$, New York J. Math. 18 (2012), 463-477.

[22] A. J. Izzo, No topological condition implies equality of polynomial and rational hulls, Proc. Amer. Math. Soc. 147 (2019), no. 12, 5195-5207.

[23] A. J. Izzo and E. L. Stout, Hulls of surfaces, Indiana Univ. Math. J. 67 (2018), no. 5, 2061-2087.

[24] S. O. Kochman, Bordism, stable homotopy and Adams spectral sequences, Fields Inst. Monogr. 7, American Mathematical Society, Providence 1996.

[25] E. L $\phi w$ and E. F. Wold, Polynomial convexity and totally real manifolds, Complex Var. Elliptic Equ. 54 (2009), no. 3-4, 265-281.

[26] S. Minsker, Some applications of the Stone-Weierstrass theorem to planar rational approximation, Proc. Amer. Math. Soc. 58 (1976), 94-96.

[27] S. Nemirovski and K. Siegel, Rationally convex domains and singular Lagrangian surfaces in $\mathbb{C}^{2}$, Invent. Math. 203 (2016), no. 1, 333-358. 
[28] A. G. O'Farrell, K. J. Preskenis and D. Walsh, Holomorphic approximation in Lipschitz norms, in: Proceedings of the conference on Banach algebras and several complex variables (New Haven 1983), Contemp. Math. 32, American Mathematical Society, Providence (1984), 187-194.

[29] G. Stolzenberg, Polynomially and rationally convex sets, Acta Math. 109 (1963), 259-289.

[30] E. L. Stout, Polynomial convexity, Progr. Math. 261, Birkhäuser, Boston 2007.

[31] D. G. Vodovoz and M. G. Zaidenberg, The number of generators in an algebra of continuous functions, Math. Notes 10 (1971), 746-748.

[32] R. O. Wells, Jr., Compact real submanifolds of a complex manifold with nondegenerate holomorphic tangent bundles, Math. Ann. 179 (1969), 123-129.

Purvi Gupta, Department of Mathematics, Indian Institute of Science,

Bangalore 560012, India

e-mail: purvigupta@iisc.ac.in

Rasul Shafikov, Department of Mathematics, University of Western Ontario,

London, Ontario N6A 5B7, Canada

e-mail:shafikov@uwo.ca

Eingegangen 23. Oktober 2020, in revidierter Fassung 14. März 2021 